Pure and Applied Mathematics Quarterly

Volume 10, Number 2

(Special Issue: In memory of

Andrey Todorov, Part 3 of 3 )

$193-222,2014$

\title{
Irreducible Components of Hurwitz Spaces Parameterizing Galois Coverings of Curves of Positive Genus
}

\author{
Vassil Kanev \\ To the memory of my friend and teacher, \\ Andrey Todorov
}

\begin{abstract}
Given a smooth, projective curve $Y$ of genus $\geq 1$ and a finite group $G$, let $H_{n}^{G}(Y)$ be the Hurwitz space which parameterizes the $G$-equivalence classes of $G$-coverings of $Y$ branched in $n$ points. This space is a finite étale covering of $Y^{(n)} \backslash \Delta$, where $\Delta$ is the big diagonal. In this paper we calculate explicitly the monodromy of this covering. This is an extension to curves of positive genus of a well known result in the case of $Y \cong \mathbb{P}^{1}$, and may be used for determining the irreducible components of $H_{n}^{G}(Y)$ in particular cases.
\end{abstract}

Keywords: Hurwitz space, Galois covering, braid group.

\section{INTRODUCTION}

Let $Y$ be a smooth, projective, irreducible complex curve. A $G$-covering $p: C \rightarrow Y$ is a Galois covering, where $C$ is a smooth, projective, irreducible curve and an isomorphism $G \stackrel{\sim}{\longrightarrow} A u t(C / Y)$ is fixed. Two $G$-coverings are equivalent if there is a $G$-equivariant isomorphism between them. We are concerned with the

Received December 16, 2012.

2010 Mathematics Subject Classification. Primary 14H10; Secondary 14H30, 20F36

This work was supported by a research grant from the University of Palermo. 
Hurwitz spaces $H_{n}^{G}(Y)$ and $H_{n}^{G}\left(Y, y_{0}\right)$. The first one parameterizes $G$-equivalence classes of $G$-coverings of $Y$ branched in $n$ points. The second one, given a point $y_{0} \in Y$, parameterizes $G$-equivalence classes of pairs $\left[p: C \rightarrow Y, z_{0}\right]$, where $p: C \rightarrow Y$ is a $G$-covering unramified at $y_{0}$ and $z_{0} \in p^{-1}\left(y_{0}\right)$. When $G=S_{d}$ one can equivalently consider coverings $f: X \rightarrow Y$ of degree $d$ with full monodromy group $S_{d}$. The Hurwitz spaces are smooth algebraic varieties and associating to a covering its branch divisor yields finite étale morphisms $H_{n}^{G}(Y) \rightarrow Y^{(n)}-\Delta$ and $H_{n}^{G}(Y) \rightarrow\left(Y-y_{0}\right)^{(n)}-\Delta$, where $\Delta$ is the codimension one subvariety of $Y^{(n)}$ whose points correspond to effective non simple divisors of $Y$.

The main result of the present paper is the explicit calculation, when $g(Y) \geq 1$, of the monodromy action of the fundamental groups of $Y^{(n)}-\Delta$ and $\left(Y-y_{0}\right)^{(n)}-\Delta$ on the fibers of the above topological coverings (see Theorem 2.8 and Theorem 2.10). The connected (=irreducible) components of $H_{n}^{G}(Y)$ and $H_{n}^{G}\left(Y, y_{0}\right)$ are in one-to-one correspondence with the orbits of these monodromy actions.

The case $Y=\mathbb{P}^{1}, G=S_{d}$ is classical. Hurwitz, using results of Clebsch [Cl], proved in $[\mathrm{Hu}]$ the connectedness of the space which parameterizes equivalence classes of simple coverings of $\mathbb{P}^{1}$ branched in $n$ points (see [Vo2, Lemma 10.15] for a modern account). The Hurwitz spaces of Galois coverings of $\mathbb{P}^{1}$ were first introduced and studied by Fried in $[\mathrm{Fr}]$ in connection with the inverse Galois problem. Fried and Völklein prove in $[\mathrm{FV}]$ that $H_{n}^{G}\left(\mathbb{P}^{1}\right)$ has a structure of algebraic variety over $\mathbb{Q}$ and if furthermore the center of $G$ is trivial they relate the solution of the inverse Galois problem to the existence of $Q$-rational points of $H_{n}^{G}\left(\mathbb{P}^{1}\right)$. They also address the problem of determining the connected components of the complex variety $H_{n}^{G}\left(\mathbb{P}^{1}\right)$. Berstein and Edmonds study in [BE] the Hurwitz spaces of simply branched coverings $X \rightarrow Y$ and address the problem of its connectedness in relation to the topological classification of the generic branched coverings between two compact, oriented real surfaces. Harris, Graber and Starr proved in [HGS] the irreducibility of the Hurwitz space of simply branched coverings of degree $d$ of a curve of positive genus, with monodromy group $S_{d}$, branched in $\geq 2 d$ points. This result was used in the proof of their theorem on existence of sections of morphisms $V \rightarrow Y$ with rationally connected fibers. In our paper [K2] the irreducibility of this Hurwitz space was used in the proof of the unirationality of certain types of Hurwitz spaces of coverings of degree $\leq 5$ of a curve $Y$ of positive genus, thus extending to curves $Y$ of arbitrary genus a well 
known result of Arbarello and Cornalba for coverings of $\mathbb{P}^{1}$. Catanese, Lönne and Perroni achieved in [CLP] the classification of the irreducible components of the locus (in the moduli space of curves) of curves admitting an effective action of the dihedral group $G=D_{m}$. Identifying certain connected components of $H_{n}^{G}(Y)$, when $G=D_{m}$, is a part of their study.

The present paper is essentially an adaptation to the case of Galois coverings of the first two sections of the unpublished preprint [K1]. In the remaining sections of this preprint we addressed the problem of proving the irreducibility of the Hurwitz spaces of coverings $X \rightarrow Y$ of degree $d$ with full monodromy group $S_{d}$ in the cases of simple branching or simple branching in all but one point. In particular, fixing the type of the local monodromy at one special point, we obtained the irreducibility provided the number of points of $Y$ of simple branching is $\geq 2 d-2$. The lower bound $2 d-2$ was improved in the paper of F. Vetro [Ve1]. In a series of papers $F$. Vetro addressed the problem of determining the irreducible components of the Hurwitz spaces of coverings $X \rightarrow Y$ of a curve $Y$ of positive genus, imposing various conditions on the type of branching. She also studied coverings with a covering involution, when the monodromy group is not the full symmetric group, but a Weyl group of type $B_{\ell}$ or of type $D_{\ell}$ (see [Ve1, Ve2, Ve3, Ve4]). She has used in an essential way the formulas and the results of the first two sections of the preprint [K1] which we include in the present paper.

In [KuKh] Kulikov and Kharlamov used an algebraic tool called semigroup over a group in order to determine the connected components of the Hurwitz spaces of coverings of a curve of arbitrary genus with fixed type of branching and fixed monodromy group. When the number of branch points of each of the branching types is sufficiently large, they reduce the problem of finding the number of the irreducible components of the Hurwitz space to a combinatorial problem involving a certain graph.

The paper is organized as follows. In Section 1 we define and give some basic properties of the Hurwitz spaces $H_{n}^{G}(Y)$ and $H_{n}^{G}\left(Y, y_{0}\right)$. This material is well known when $Y \cong \mathbb{P}^{1}$ (cf. [FV], [Vo1], [Vo2], [Em]) and the extension to curves $Y$ of arbitrary genus is immediate. We include a detailed account for reader's convenience. 
Section 2 is devoted to the explicit calculation of the monodromy action of the braid group $\pi_{1}\left(\left(Y-y_{0}\right)^{(n)}-\Delta, D\right)$ on the fiber of $H_{n}^{G}\left(Y, y_{0}\right) \rightarrow\left(Y-y_{0}\right)^{(n)}-\Delta$ over a point $D$. The fiber is identified with the set of Hurwitz systems of $G$. These are ordered sets of elements $\left(t_{1}, \ldots, t_{n} ; \lambda_{1}, \mu_{1}, \ldots, \lambda_{g}, \mu_{g}\right)$ of $G$, which generate $G$, $t_{i} \neq 1 \forall i$ and $t_{1} t_{2} \cdots t_{n}=\left[\lambda_{1}, \mu_{1}\right] \cdots\left[\lambda_{g}, \mu_{g}\right]$. After recalling a result of Birman [Bi] which yields a system of generators of $\pi_{1}\left(\left(Y-y_{0}\right)^{(n)}-\Delta, D\right)$ we give in Theorem 2.8 explicit formulas for the monodromy action of these generators on the set of Hurwitz systems. The monodromy action for the covering $H_{n}^{G}(Y) \rightarrow$ $Y^{(n)}-\Delta$ is obtained by passing from Hurwitz systems to equivalence classes of Hurwitz systems with respect to inner automorphisms of $G$.

In Section 3 we first discuss how to extend the arguments of the previous sections removing the assumption that $G$ is finite. In Proposition 3.1 (this is the Main Lemma of [K1]) we prove a statement which turns out to be useful in determining the connected components of the Hurwitz spaces. Namely, given a Hurwitz system $\left(t_{1}, \ldots, t_{n} ; \lambda_{1}, \mu_{1}, \ldots, \lambda_{g}, \mu_{g}\right)$ of a group $G$, such that $t_{i} t_{i+1}=1$, let $H$ be the subgroup of $G$ generated by $t_{1}, \ldots, t_{i-1}, t_{i+2}, \ldots, t_{n}, \lambda_{1}, \mu_{1}, \ldots, \lambda_{g}, \mu_{g}$. Let $h \in H$. Then the given Hurwitz system and the one obtained by replacing the pair $\left(t_{i}, t_{i+1}\right)$ by $\left(h^{-1} t_{i} h, h^{-1} t_{i+1} h\right)$ belong to the same $\pi_{1}\left(\left(Y-y_{0}\right)^{(n)}-\Delta, D\right)$-orbit.

Notation and conventions. We assume that the base field $k=\mathbb{C}$. By homotopy of arcs we always mean homotopy which fixes the end points. If $G$ is a group and $t, h \in G$ we denote $h^{-1} t h$ by $t^{h}$ and $h t h^{-1}$ by ${ }^{h} t$

\section{Preliminaries on Hurwitz spaces}

In this section $Y$ is a fixed smooth, projective, irreducible curve of arbitrary genus.

1.1. We consider the product in a fundamental group induced by product of arcs defined as

$$
\gamma_{1} * \gamma_{2}= \begin{cases}\gamma_{1}(2 t) & \text { for } 0 \leq t \leq 1 / 2 \\ \gamma_{2}(2 t-1) & \text { for } 1 / 2 \leq t \leq 1\end{cases}
$$

We recall some standard facts ([Ma, Chapter 5], [Fo, Chapter 1]). Let $f: X \rightarrow Y$ be a surjective morphism of smooth, projective, irreducible curves. Let $D \subset Y$ be the branch locus. Let $Y^{\prime}=Y-D$ and $X^{\prime}=f^{-1}\left(Y^{\prime}\right)$. Then the restriction $f^{\prime}: X^{\prime} \rightarrow Y^{\prime}$ is a topological (unramified) covering. Let $y_{0} \in Y^{\prime}$. Then $\pi_{1}\left(Y^{\prime}, y_{0}\right)$ 
acts on the right on $f^{-1}\left(y_{0}\right)$ as follows: if $[\alpha] \in \pi_{1}\left(Y^{\prime}, y_{0}\right)$ and $x \in f^{-1}\left(y_{0}\right)$, let $\tilde{\alpha}_{x}: I \rightarrow X^{\prime}$ be the lifting of $\alpha$ with initial point $x$; then $x \cdot[\alpha]:=\tilde{\alpha}_{x}(1)$.

Consider the particular case of a Galois covering $p: C \rightarrow Y$ whose Galois group is isomorphic to $G$. Let us fix an isomorphism $C \stackrel{\sim}{\longrightarrow} A u t(C / Y) \cong \operatorname{Deck}\left(C^{\prime} / Y^{\prime}\right)$. Here $G$ acts on $C$ on the left. We call such a data a $G$-covering of $Y$. Let $z_{0} \in p^{-1}\left(y_{0}\right)$. Then a surjective homomorphism $m_{z_{0}}: \pi_{1}\left(Y^{\prime}, y_{0}\right) \rightarrow G$, called monodromy homomorphism is defined as follows:

$$
m_{z_{0}}([\alpha])=g \quad \text { iff } \quad z_{0} \cdot[\alpha]=g z_{0} .
$$

One has $\operatorname{Ker}\left(m_{z_{0}}\right)=p_{*}\left(\pi_{1}\left(C^{\prime}, z_{0}\right)\right)$. Suppose $p: C \rightarrow Y$ is branched in $n \geq 1$ points. Let $D=\left\{b_{1}, \ldots, b_{n}\right\}$ be the branch locus. Let us choose neighborhoods $U_{i} \ni b_{i}$ such that every $\bar{U}_{i}$ is homeomorphic to the unit disk, $i=1, \ldots, n$, and $\bar{U}_{i} \cap \bar{U}_{j}=\emptyset$ for $\forall i \neq j$. For each $i=1, \ldots, n$ let us choose an $\operatorname{arc} \eta_{i}$ in $Y-\cup_{i=1}^{n} U_{i}$ such that $\eta_{i}(0)=y_{0}, \eta_{i}(1) \in \partial \bar{U}_{i}$, then let $\gamma_{i}$ be the closed arc which starts at $y_{0}$, travels along $\eta_{i}$, makes a counterclockwise loop along $\partial \bar{U}_{i}$ and returns to $y_{0}$ along $\eta_{i}^{-}$. It is clear that $m_{z_{0}}\left(\left[\gamma_{i}\right]\right)$, called local monodromy at $b_{i}$ along $\gamma_{i}$, has the property $m_{z_{0}}\left(\left[\gamma_{i}\right]\right) \neq 1$ for every $i=1, \ldots, n$. Let $O_{i} \subset G$ be the conjugacy class of $m_{z_{0}}\left(\left[\gamma_{i}\right]\right)$. Another choice of $U_{i}$ and $\eta_{i}$ as above results in replacing each $m_{z_{0}}\left(\left[\gamma_{i}\right]\right)$ by another element in $O_{i}$. We conclude that $m=m_{z_{0}}: \pi_{1}\left(Y^{\prime}, y_{0}\right) \rightarrow G$ satisfies the following condition:

$$
m\left(\left[\gamma_{1}\right]\right) \neq 1, \ldots, m\left(\left[\gamma_{n}\right]\right) \neq 1
$$

which is furthermore independent of the choice of $\gamma_{i}, i=1, \ldots, n$ as above.

1.2. Two $G$-coverings $p: C \rightarrow Y$ and $p_{1}: C_{1} \rightarrow Y$ are called $G$-equivalent if there is a $G$-equivariant isomorphism $f: C \rightarrow C_{1}$ such that $p_{1}=f \circ p$. Fixing $y_{0} \in Y^{\prime}$ let $z_{0} \in p^{-1}\left(y_{0}\right), z_{1} \in p_{1}^{-1}\left(y_{0}\right)$. The pairs $\left(C, z_{0}\right),\left(C_{1}, z_{1}\right)$ are called $G$-equivalent if there is an isomorphism $f$ as above, which furthermore satisfies $f\left(z_{0}\right)=z_{1}$. Such an $f$ is unique, when exists, since every covering automorphism of $C^{\prime} \rightarrow Y^{\prime}$ which fixes $z_{0}$ is the identity. It is clear that if $\left(C, z_{0}\right)$ and $\left(C_{1}, z_{1}\right)$ are $G$-equivalent, then $m_{z_{0}}=m_{z_{1}}$.

Proposition 1.3 (Riemann's Existence Theorem). Let $D \subset Y$ be a finite set, $|D|=n$. Let $Y^{\prime}=Y-D$ and let $y_{0} \in Y^{\prime}$. Let $G$ be a finite group. The following sets are in one-to-one correspondence:

(a) the set of $G$-equivalence classes of pairs $\left(p: C \rightarrow Y, z_{0}\right)$, where $p: C \rightarrow Y$ is a $G$-covering, whose branch locus equals $D$ and $z_{0} \in p^{-1}\left(y_{0}\right)$; 
(b) the set of epimorphisms $m: \pi_{1}\left(Y^{\prime}, y_{0}\right) \rightarrow G$, which satisfy Condition 3. If two pairs $\left(p: C \rightarrow Y, z_{0}\right)$ and $\left(p_{1}: C_{1} \rightarrow Y, z_{1}\right)$ correspond to one and the same $m: \pi_{1}\left(Y^{\prime}, y_{0}\right) \rightarrow G$, then the $G$-equivalence $f: C \rightarrow C_{1}, f\left(z_{0}\right)=z_{1}$ is unique.

Proof. One associates to $\left(p: C \rightarrow Y, z_{0}\right)$ as in (a) the epimorphism $m=m_{z_{0}}$. The statements of the proposition are standard (see [Fo] and [Vo2]). We only mention how one constructs a $G$-covering from an epimorphism $m: \pi_{1}\left(Y^{\prime}, y_{0}\right) \rightarrow G$. Let $\Gamma=\operatorname{Ker}(m)$. One lets

$$
C^{\prime}=\left\{\Gamma[\alpha] \mid[\alpha] \text { is a homotopy class of arcs with } \alpha(0)=y_{0}, \alpha(1) \in Y^{\prime}\right\} .
$$

The map $p^{\prime}: C^{\prime} \rightarrow Y^{\prime}$ is defined by $p^{\prime}(\Gamma[\alpha])=\alpha(1)$. If $g \in G$ and $m([\sigma])=g$ one lets for $z=\Gamma[\alpha] \in C^{\prime}$

$$
g z=\Gamma[\sigma * \alpha] .
$$

One defines in a natural way topology on $C^{\prime}$ such that $p^{\prime}: C^{\prime} \rightarrow Y^{\prime}$ is a topological covering map. It is regular and $G \cong \operatorname{Deck}\left(C^{\prime} / Y^{\prime}\right)$. The complex analytic structure on $Y^{\prime}$ induces a complex analytic structure on $C^{\prime}$. Compactifying one obtains a holomorphic map of compact Riemann surfaces $p: C \rightarrow Y$ branched at $D$ and the action of $G$ on $C^{\prime}$ extends to an action on $C$. The distinguished point $z_{0}$ is $\Gamma\left[c_{y_{0}}\right]$, where $c_{y_{0}}: I \rightarrow\left\{y_{0}\right\} \subset Y^{\prime}$. One has $m_{z_{0}}=m$.

It remains to prove that $p: C \rightarrow Y$ has an algebraic structure. Let $K=\mathcal{M}(C)$ be the field of meromorphic functions of $C$. It is a Galois extension of the field of rational functions $\mathbb{C}(Y)$ with group isomorphic to $G$ (see [Vo2, Theorem 5.9], where $Y \cong \mathbb{P}^{1}$ but the arguments apply to $Y$ of arbitrary genus). Let $f: X \rightarrow Y$ be the normalization of $Y$ in $K$. One has $\mathcal{M}\left(X^{a n}\right)=\mathbb{C}(X) \cong K=\mathcal{M}(C)$. Choosing a primitive element $F$ of the field extension $K / \mathbb{C}(Y)$ and using [Fo, Proposition 8.9] one concludes that $p: C \rightarrow Y$ is biholomorphically equivalent to $f^{a n}: X^{a n} \rightarrow Y$. Furthermore this equivalence is $G$-equivariant and compatible with the action of $G$ on the field $K$ as follows from [Fo, Proposition 8.12].

Let $Y^{(n)}$ be the symmetric product of $Y$ with itself $n$ times and let $\Delta \subset Y^{(n)}$ be the codimension one subvariety whose elements are the effective divisors of degree $n$ which have points of multiplicity $>1$. Abusing notation we identify a point $D \in Y^{(n)}-\Delta, \quad D=b_{1}+\cdots+b_{n}$ with its support $\operatorname{Supp}(D)=\left\{b_{1} \ldots, b_{n}\right\}$. 
Definition 1. Let $y_{0} \in Y$. Let $G$ be a finite group. We denote by $H_{n}^{G}\left(Y, y_{0}\right)$ the set of pairs $(D, m)$ where $D \in\left(Y-y_{0}\right)^{(n)}-\Delta$ and $m: \pi_{1}\left(Y-D, y_{0}\right) \rightarrow G$ is a surjective homomorphism which satisfies Condition 3.

According to Proposition 1.3 the set $H_{n}^{G}\left(Y, y_{0}\right)$ is in bijective correspondence with the set of equivalence classes $\left[p: C \rightarrow Y, z_{0}\right.$ ], where $p: C \rightarrow Y$ is a $G$-covering branched in $n$ points, unramified at $y_{0}, z_{0} \in p^{-1}\left(y_{0}\right)$ and the equivalence is the $G$-equivalence as defined in $\S 1.2$.

Example. Let $f: X \rightarrow Y$ be a covering of smooth, projective, irreducible curves of degree $d$ with branch locus $D,|D|=n$. Let $y_{0} \notin D$. Let $\Lambda=f^{-1}\left(y_{0}\right)$, let $S(\Lambda)$ be the symmetric group acting on the right on $\Lambda$. Suppose the monodromy homomorphism $\mu: \pi_{1}\left(Y-D, y_{0}\right) \rightarrow S(\Lambda)$ is surjective. The associated Galois covering is explicitly constructed as follows. The curve

$$
C^{\prime}=\left\{\left(x_{1}, \ldots, x_{n}\right) \mid\left\{x_{1}, \ldots, x_{n}\right\}=f^{-1}(y), y \in Y-D\right\}
$$

is an irreducible, finite, étale covering of $Y-D$ of degree $n !$. The group $S_{d}$ of permutations is considered acting on the right on $\{1, \ldots, d\}$. For $\sigma \in S_{d}$ we write $i^{\sigma}$ for the image of $i$. The group $S_{d}$ acts on the left on $C^{\prime}$ by $\sigma$. $\left(x_{1}, \ldots, x_{d}\right)=\left(x_{1^{\sigma}}, \ldots, x_{d^{\sigma}}\right)$. The smooth completion $p: C \rightarrow Y$ is the Galois covering associated with $f: X \rightarrow Y$. To fix a point $z_{0} \in C, z_{0} \in p^{-1}\left(y_{0}\right)$ means here to fix a bijection $\phi: f^{-1}\left(y_{0}\right) \rightarrow\{1, \ldots, d\}$.

Let $p: C \rightarrow Y$ be a $G$-covering with branch locus $D$. Let $Y^{\prime}=Y-D$. Let $y_{0}, y_{1} \in Y^{\prime}$, let $z_{0} \in p^{-1}\left(y_{0}\right), z_{1} \in p^{-1}\left(y_{1}\right)$. Let us connect $z_{0}$ and $z_{1}$ by an arc in $C^{\prime}=p^{-1}\left(Y^{\prime}\right)$ and let $\tau$ be its projection in $Y^{\prime}$. Then one has the following relation between $m_{z_{0}}: \pi_{1}\left(Y^{\prime}, y_{0}\right) \rightarrow G$ and $m_{z_{1}}: \pi_{1}\left(Y^{\prime}, y_{1}\right) \rightarrow G$ :

$$
m_{z_{0}}\left(\left[\tau * \alpha * \tau^{-}\right]\right)=m_{z_{1}}([\alpha]) \quad \text { for every } \quad[\alpha] \in \pi_{1}\left(Y^{\prime}, y_{1}\right)
$$

Definition 2. Let $y_{0}, y_{1} \in Y^{\prime}=Y-D$. Two homomorphisms $m_{0}: \pi_{1}\left(Y^{\prime}, y_{0}\right) \rightarrow$ $G$ and $m_{1}: \pi_{1}\left(Y^{\prime}, y_{1}\right) \rightarrow G$ are called arcwise connected if there is an $\operatorname{arc} \tau$ : $I \rightarrow Y^{\prime}$ with $\tau(0)=y_{0}, \tau(1)=y_{1}$ such that $m_{1}([\alpha])=m_{0}\left(\left[\tau * \alpha * \tau^{-}\right]\right)$for every $[\alpha] \in \pi_{1}\left(Y^{\prime}, y_{1}\right)$. We write $m_{1}=m_{0}^{\tau}$.

1.4. The following statements are easily verified.

(i) Arcwise connectedness is a relation of equivalence. 
(ii) Given a $G$-covering $p: C \rightarrow Y$ and $m_{z_{0}}: \pi_{1}\left(Y^{\prime}, y_{0}\right) \rightarrow G$ as in $\S 1.1$ every $m_{1}: \pi_{1}\left(Y^{\prime}, y_{1}\right) \rightarrow G$ arcwise connected with $m_{z_{0}}$ equals $m_{z_{1}}$ for some $z_{1} \in p^{-1}\left(Y^{\prime}\right)$.

(iii) Given a surjective homomorphism $m: \pi_{1}\left(Y^{\prime}, y_{0}\right) \rightarrow G$, a homomorphism $m_{1}: \pi_{1}\left(Y^{\prime}, y_{0}\right) \rightarrow G$ is arcwise connected with $m$ if and only if it belongs to the orbit ${ }^{G} m=\left\{g m g^{-1} \mid g \in G\right\}$ where $g m g^{-1}([\alpha])=g m([\alpha]) g^{-1}$ for every $[\alpha] \in \pi_{1}\left(Y^{\prime}, y_{0}\right)$.

(iv) If $m: \pi_{1}\left(Y^{\prime}, y_{0}\right) \rightarrow G$ satisfies Condition 3 then every $m_{1}: \pi_{1}\left(Y^{\prime}, y_{1}\right) \rightarrow G$ arcwise connected with $m$ satisfies it.

Proposition 1.5. Let $D \subset Y$ be a finite set, $|D|=n$. Let $Y^{\prime}=Y-D$, let $y_{0} \in$ $Y^{\prime}$. Let $G$ be a finite group. The following sets are in one-to-one correspondence:

(a) the set of $G$-equivalence classes of $G$-coverings $p: C \rightarrow Y$ whose branch locus equals $D$.

(b) the set of arcwise connected families of epimorphisms $\underline{m}=\left\{m: \pi_{1}\left(Y^{\prime}, y\right) \rightarrow\right.$ $\left.G \mid y \in Y^{\prime}\right\}$ which satisfy Condition 3

Suppose the center of the group is trivial: $Z(G)=1$. In this case if two coverings $p: C \rightarrow Y$ and $p_{1}: C_{1} \rightarrow Y$ correspond to one and the same $\underline{m}$, then the G-equivalence $f: C \rightarrow C_{1}$ is unique. This property holds if and only if $Z(G)=1$.

Proof. Given $p: C \rightarrow Y$ as in (a) one lets $\underline{m}=\left\{m_{z}: \pi_{1}\left(Y^{\prime}, y\right) \rightarrow G \mid p(z)=y \in\right.$ $\left.Y^{\prime}\right\}$. The proposition follows from: Proposition 1.3; the statements of $\S 1.4$; the fact that every covering automorphism of $p: C \rightarrow Y$ is of the form $\sigma(z)=h z$ for some $h \in G$ and from the fact that it is $G$-equivariant iff $h \in Z(G)$.

Definition 3. Let $G$ be a finite group. We denote by $H_{n}^{G}(Y)$ the set of pairs $(D, \underline{m})$, where $D \in Y^{(n)}-\Delta$ and $\underline{m}$ is a family of arcwise connected epimorphisms $m: \pi_{1}(Y-D, y) \rightarrow G$, which satisfy Condition 3 .

1.6. According to Proposition 1.5 the set $H_{n}^{G}(Y)$ is in bijective correspondence with the set of equivalence classes $[p: C \rightarrow Y]$, where $p: C \rightarrow Y$ is a $G$-covering branched in $n$ points with respect to the $G$-equivalence defined in $\S 1.2$. Let $y_{0} \in Y$ and let $U\left(y_{0}\right) \subset H_{n}^{G}(Y)$ be the subset $\left\{(D, \underline{m}) \mid y_{0} \notin D\right\}$. By $\S 1.4$ (iii) the map $H_{n}^{G}\left(Y, y_{0}\right) \rightarrow U\left(y_{0}\right)$ given by $(D, m) \mapsto(D, \underline{m})$ is $G$-invariant with respect to the action $g(D, m)=\left(D, g m g^{-1}\right)$ and $U\left(y_{0}\right)$ is bijective to $H_{n}^{G}\left(Y, y_{0}\right) / G$. 
1.7. Let $b: H_{n}^{G}\left(Y, y_{0}\right) \rightarrow\left(Y-y_{0}\right)^{(n)}-\Delta$ be the map $b(D, m)=D$. Following [Fu, $\S 1.3]$ we want to define a Hausdorff topology on $H_{n}^{G}\left(Y, y_{0}\right)$ which makes $b$ a topological covering map. Let $(D, m) \in H_{n}^{G}\left(Y, y_{0}\right)$. Let $D=b_{1}+\cdots+b_{n}$. Let $U_{i} \ni b_{i}$ be neighborhoods, such that every $\bar{U}_{i}$ is homeomorphic to the unit disk, $i=1, \ldots, n$, furthermore $\bar{U}_{1}, \ldots, \bar{U}_{n}$ are disjoint and $y_{0} \notin \cup_{i=1}^{n} \bar{U}_{i}$. Let $N\left(U_{1}, \ldots, U_{n}\right) \subset\left(Y-y_{0}\right)^{(n)}-\Delta$ be the neighborhood of $D$ consisting of $E=$ $y_{1}+\cdots+y_{n}$ with $y_{i} \in U_{i}$. Let $U=\cup_{i=1}^{n} U_{i}$. The inclusion $Y-U \rightarrow Y-D$ is a deformation retract, so for every epimorphism $m: \pi_{1}\left(Y-D, y_{0}\right) \rightarrow G$ there is a unique epimorphism $m(E): \pi_{1}\left(Y-E, y_{0}\right) \rightarrow G$ such that the following diagram commutes

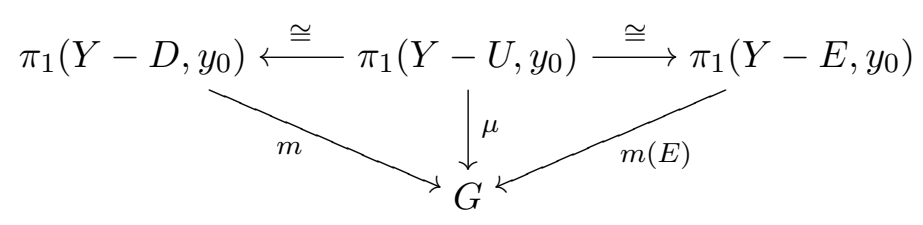

We let

$$
N_{m}\left(U_{1}, \ldots, U_{n}\right)=\left\{(E, m(E)) \mid E \in N\left(U_{1}, \ldots, U_{n}\right\}\right.
$$

Proposition 1.8. Let $Y$ be a smooth, projective, irreducible curve. Let $y_{0} \in Y$. Let $G$ be a finite group. Let $H_{n}^{G}\left(Y, y_{0}\right)$ be as in Definition 1. Suppose $H_{n}^{G}\left(Y, y_{0}\right) \neq$ $\emptyset$.

(a) $H_{n}^{G}\left(Y, y_{0}\right)$ may be endowed by a Hausdorff topology such that the map $b: H_{n}^{G}\left(Y, y_{0}\right) \rightarrow\left(Y-y_{0}\right)^{(n)}-\Delta$ given by $b(D, m)=D$ is a covering map. A basis of open sets of this topology is the family of sets $N_{m}\left(U_{1}, \ldots, U_{n}\right)$ obtained varying $D \in\left(Y-y_{0}\right)^{(n)}-\Delta, m: \pi_{1}\left(Y-D, y_{0}\right) \rightarrow G$ and $U_{1}, \ldots, U_{n}$ as above. Every open set $N\left(U_{1}, \ldots, U_{n}\right) \subset\left(Y-y_{0}\right)^{(n)}-\Delta$ is evenly covered: $b^{-1}\left(N\left(U_{1}, \ldots, U_{n}\right)\right)$ is a disjoint union of open sets homeomorphic to $N\left(U_{1}, \ldots, U_{n}\right)$. The connected components of $b^{-1}\left(N\left(U_{1}, \ldots, U_{n}\right)\right)$ are in bijective correspondence with the set of epimorphisms $\mu: \pi_{1}\left(Y-\cup_{i=1}^{n} U_{i}, y_{0}\right) \rightarrow G$. The covering $b$ is proper.

(b) $H_{n}^{G}\left(Y, y_{0}\right)$ has a unique structure of a complex manifold, such that $b: H_{n}^{G}\left(Y, y_{0}\right) \rightarrow\left(Y-y_{0}\right)^{(n)}-\Delta$ is holomorphic. Furthermore $b$ is a finite étale holomorphic map.

(c) $H_{n}^{G}\left(Y, y_{0}\right)$ has a unique structure of a $\mathbb{C}$-scheme of finite type, such that $H_{n}^{G}\left(Y, y_{0}\right)^{a n}$ is biholomorphic to the complex manifold of Part (b). Furthermore $H_{n}^{G}\left(Y, y_{0}\right)$ is smooth, equidimensional of dimension $n$ and $b: H_{n}^{G}\left(Y, y_{0}\right) \rightarrow$ $\left(Y-y_{0}\right)^{(n)}-\Delta$ is a finite, étale morphism. 
(d) $H_{n}^{G}\left(Y, y_{0}\right)$ is an affine variety.

Proof. The statements of (a) are easily verified and are left to the reader.

(b) Starting from a complex analytic atlas of $\left(Y-y_{0}\right)^{(n)}-\Delta$ with charts contained in various $N\left(U_{1}, \ldots, U_{n}\right)$ one constructs a complex analytic atlas of $H_{n}^{G}\left(Y, y_{0}\right)$ and with this complex analytic structure the map $b: H_{n}^{G}\left(Y, y_{0}\right) \rightarrow$ $\left(Y-y_{0}\right)^{(n)}-\Delta$ is holomorphic, finite and étale. Let $M$ be this complex manifold. If $M^{\prime}$ is another complex manifold with underlying topological space $H_{n}^{G}\left(Y, y_{0}\right)$, such that $b: M^{\prime} \rightarrow\left(Y-y_{0}\right)^{(n)}-\Delta$ is holomorphic, then every $x \in M^{\prime}$ has a complex analytic chart $(V, \phi), \phi(V) \subset \mathbb{C}^{n}$, such that $b$ maps $V$ homeomorphically in some $N\left(U_{1}, \ldots, U_{n}\right)$. It is known that such a map $b: V \rightarrow b(V)$ is biholomorphic (cf. [Nar, p. 86]). Therefore $i d: M \rightarrow M^{\prime}$ is biholomorphic.

(c) These statements follow from Théorèm 5.1, Proposition 3.1 and Proposition 3.2 of [SGA1, Exposé XII].

(d) The curve $Y-y_{0}$ is affine, so $\left(Y-y_{0}\right)^{(n)}-\Delta$ is an affine variety. Therefore the finite cover $H_{n}^{G}\left(Y, y_{0}\right)$ is an affine variety.

Proposition 1.9. Let $Y$ be a smooth, projective, irreducible curve. Let $G$ be a finite group. Let $H_{n}^{G}(Y)$ be the set of Definition 3. Suppose $H_{n}^{G}(Y) \neq \emptyset$.

(a) $H_{n}^{G}(Y)$ may be endowed by a Hausdorff topology such that the map $b$ : $H_{n}^{G}(Y) \rightarrow Y^{(n)}-\Delta$ given by $b(D, \underline{m})=D$ is a proper covering map. For every $D \in Y^{(n)}-\Delta$ any neighborhood $N\left(U_{1}, \ldots, U_{n}\right)$ (cf. $\left.\S 1.7\right)$ is evenly covered.

(b) $H_{n}^{G}(Y)$ has a unique structure of a complex manifold such that $b: H_{n}^{G}(Y) \rightarrow$ $Y^{(n)}-\Delta$ is holomorphic. Furthermore $b$ is a finite étale holomorphic map.

(c) $H_{n}^{G}(Y)$ has a unique structure of a $\mathbb{C}$-scheme of finite type, such that $H_{n}^{G}(Y)^{a n}$ is biholomorphic to the complex manifold of Part (b). Furthermore $H_{n}^{G}(Y)$ is smooth, equidimensional of dimension $n$ and $b: H_{n}^{G}(Y) \rightarrow Y^{(n)}-\Delta$ is a finite, étale morphism.

(d) $H_{n}^{G}(Y)$ is a quasiprojective variety. If $Y \cong \mathbb{P}^{1}$ it is an affine variety.

Proof. (a) Let $y_{0} \in Y$. The group $G$ acts on $H_{n}^{G}\left(Y, y_{0}\right)$ by $g(D, m)=\left(D, g m g^{-1}\right)$. This induces a faithful, properly discontinuous action of $G / Z(G)$ on $H_{n}^{G}\left(Y, y_{0}\right)$. One endows $U\left(y_{0}\right) \cong H_{n}^{G}\left(Y, y_{0}\right) / G$ by the quotient topology (cf. $\S 1.6$ ). One has that $H_{n}^{G}(Y)=\cup_{y \in Y} U(y)$ and for each pair $y_{1}, y_{2} \in Y$ the intersection $U\left(y_{1}\right) \cap$ 
$U\left(y_{2}\right)$ is open in $U\left(y_{i}\right), i=1,2$. This defines a topology on $H_{n}^{G}(Y)$. Let $D=$ $b_{1}+\cdots+b_{n} \in Y^{(n)}-\Delta$, let $U_{i} \ni b_{i}, y_{0} \notin \cup_{i=1}^{n} \bar{U}_{i}$ be as in $\S 1.7$. Let $\underline{m}=\{m$ : $\left.\pi_{1}(Y-D, y) \rightarrow G\right\}$ be a family of arcwise connected epimorphisms. It is uniquely determined by a $G$-orbit ${ }^{G} m=\left\{g m g^{-1} \mid g \in G\right\}$ for some $m: \pi_{1}\left(Y-D, y_{0}\right) \rightarrow G$ (cf. $\S 1.4)$. Passing to $G$-orbits in (4) let $\underline{m}(E)$ correspond to ${ }^{G} m(E)$. We let

$$
N_{\underline{m}}\left(U_{1}, \ldots, U_{n}\right)=\left\{(E, \underline{m}(E)) \mid E \in N\left(U_{1}, \ldots, U_{n}\right\} .\right.
$$

This is an open subset of $U\left(y_{0}\right)$ equal to the image of $N_{m}\left(U_{1}, \ldots, U_{n}\right)$ under the quotient $\operatorname{map} H_{n}^{G}\left(Y, y_{0}\right) \rightarrow U\left(y_{0}\right)$. One has the equality

$$
b^{-1}\left(N\left(U_{1}, \ldots, U_{n}\right)\right)=\bigsqcup_{\underline{m}} N_{\underline{m}}\left(U_{1}, \ldots, U_{n}\right)
$$

and furthermore for every $\underline{m}$ the restriction $b: N_{\underline{m}}\left(U_{1}, \ldots, U_{n}\right) \rightarrow N\left(U_{1}, \ldots, U_{n}\right)$ is a homeomorphism, as it follows from the corresponding statement for the covering $H_{n}^{G}\left(Y, y_{0}\right) \rightarrow\left(Y-y_{0}\right)^{(n)}-\Delta$.

The statements of (b) and (c) are proved similarly to those of Proposition 1.9.

(d) It suffices to prove that each connected component $H$ of $H_{n}^{G}(Y)$ is quasiprojective. Every such $H$ is irreducible since $H_{n}^{G}(Y)$ is nonsingular. Let $\bar{H}$ be the normalization of $Y^{(n)}$ in the field $\mathbb{C}(H)$. Then $\bar{H}$ is projective since $Y^{(n)}$ is and $H$ is isomorphic to a Zariski open subset of $\bar{H}$. Therefore $H$ is quasiprojective. If $Y \cong \mathbb{P}^{1}$, then $Y^{(n)} \cong \mathbb{P}^{n}$ and $\Delta$ is isomorphic to a hypersurface of degree $n$. So $Y^{(n)}-\Delta$ is an affine variety. Therefore $H_{n}^{G}(Y)$ is an affine variety as well.

Definition 4. Let $O_{1}, \ldots, O_{k}$ be conjugacy classes of $G, O_{i} \neq O_{j}$ if $i \neq j$. Let $\underline{n}=n_{1} O_{1}+\cdots+n_{k} O_{k}$ be a formal sum where $n_{i} \in \mathbb{N}$. Let $|\underline{n}|:=n_{1}+\cdots+n_{k}=n$. We denote by $H_{\underline{n}}^{G}\left(Y, y_{0}\right)\left(\operatorname{resp} . H_{\underline{n}}^{G}(Y)\right)$ the subset of $H_{n}^{G}\left(Y, y_{0}\right)\left(\operatorname{resp} . H_{n}^{G}(Y)\right)$ whose points correspond to $G$-equivalence classes $\left[p: C \rightarrow Y, z_{0}\right]$ (resp. $[p: C \rightarrow$ $Y]$ ) where the coverings $p: C \rightarrow Y$ have $n_{i}$ branch points with local monodromies which belong to $O_{i}, i=1, \ldots, k$.

It is clear that $H_{\underline{n}}^{G}\left(Y, y_{0}\right)$ is a union of connected components of $H_{n}^{G}\left(Y, y_{0}\right)$ if it is nonempty and one has

$$
H_{n}^{G}\left(Y, y_{0}\right)=\bigsqcup_{|\underline{n}|=n} H_{\underline{n}}^{G}\left(Y, y_{0}\right)
$$


Similarly $H_{\underline{n}}^{G}(Y)$ is a union of connected components of $H_{n}^{G}(Y)$ if it is nonempty and one has

$$
H_{n}^{G}(Y)=\bigsqcup_{|\underline{n}|=n} H_{\underline{n}}^{G}(Y)
$$

\section{BRAID MOVES}

2.1. Suppose that $g(Y) \geq 1$. Let us fix the orientation of the real 2-manifold $Y$ considered as a complex manifold. Let $D=\left\{b_{1}, \ldots, b_{n}\right\} \subset Y$. Let $b_{0} \notin D$. We describe a standard way of choosing generators of $\pi_{1}\left(Y-D, b_{0}\right)$. Cutting $Y$ along $2 g$ simple closed arcs, which begin at $b_{0}$ and do not contain any of $b_{i}, i \geq 1$, one obtains a standard $4 g$-polygon with sides $\alpha_{1}, \beta_{1}, \alpha_{1}^{-1}, \beta_{1}^{-1}, \ldots, \alpha_{g}, \beta_{g}, \alpha_{g}^{-1}, \beta_{g}^{-1}$ which circle the polygon in counterclockwise direction. We consider a simple closed arc $L$ which begins at $b_{0}, \quad L-b_{0}$ is contained in the interior of the 4 g-polygon and passes consecutively in counterclockwise direction through the points $\left\{b_{1}, \ldots, b_{n}\right\}$. The closed $\operatorname{arc} L$ divides the $4 g$-polygon into two regions $R$ and $R^{\prime}$ which stay on the left, respectively on the right side of $L$ with respect to its counterclockwise orientation. We choose a simple arc $\ell_{1}$ which lies inside the region $R$ and connects $b_{0}$ and $b_{1}$. Then we choose a second simple arc $\ell_{2}$ inside $R$ which connects $b_{0}$ with $b_{2}$, has only $b_{0}$ as point in common with $\ell_{1}$, and lies on the left side of $\ell_{1}$. Continuing in this way we obtain an ordered $n$-tuple $\left(\ell_{1} \ldots, \ell_{n}\right)$ of simple arcs which do not meet outside $b_{0}$. Let $\gamma_{i}$ be a closed arc which begins at $b_{0}$, travels along $\ell_{i}$ to a point near $b_{i}$, makes a small counterclockwise loop around $b_{i}$, and returns to $b_{0}$ along $\ell_{i}$. We obtain a $(n+2 g)$-tuple of closed $\operatorname{arcs}\left(\gamma_{1}, \ldots, \gamma_{n} ; \alpha_{1}, \beta_{1}, \ldots, \alpha_{g}, \beta_{g}\right)$ which we call a standard system of closed arcs. The corresponding homotopy classes yield a standard system of generators for $\pi_{1}\left(Y-D, b_{0}\right)$ which satisfy the only relation

$$
\gamma_{1} \gamma_{2} \cdots \gamma_{n} \simeq\left[\alpha_{1}, \beta_{1}\right] \cdots\left[\alpha_{g}, \beta_{g}\right]
$$

Figure 1 illustrates such a standard system. A reader who prefers the clockwise orientation of closed arcs and ordering of the branch points from left to right may look at this and all subsequent figures from the other side of the sheet. Here and in the rest of the paper we will use the same notation, for the sake of its simplification, for a closed arc and for its homotopy class when the correct meaning is clear from the context. Given a $G$-covering $p: C \rightarrow Y$ with branch 


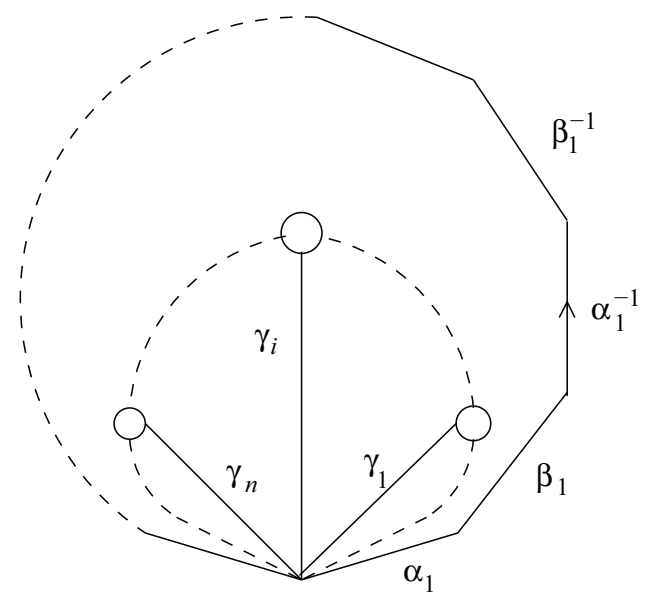

FiguRE 1. Standard system of closed arcs

locus $D$, a point $b_{0} \in Y-D$ and a point $z_{0} \in p^{-1}\left(b_{0}\right)$ consider the homomorphism $m=m_{z_{0}}: \pi_{1}\left(Y-D, b_{0}\right) \rightarrow G$. Let $t_{i}=m\left(\gamma_{i}\right), \lambda_{k}=m\left(\alpha_{k}\right), \mu_{k}=\lambda_{g+k}=m\left(\beta_{k}\right)$.

Definition 5. Let $G$ be a group. An ordered set $\left(t_{1}, \ldots, t_{n} ; \lambda_{1}, \mu_{1}, \ldots, \lambda_{g}, \mu_{g}\right)$ of elements of $G$ which generate $G$, has the property $t_{i} \neq 1$ for $\forall i$ and satisfies the relation

$$
t_{1} t_{2} \cdots t_{n}=\left[\lambda_{1}, \mu_{1}\right] \cdots\left[\lambda_{g}, \mu_{g}\right]
$$

is called a Hurwitz system (of generators) of the group $G$. We let $\lambda_{g+k}=\mu_{k}$.

According to Proposition1.3, given $D \in Y^{(n)}-\Delta$ and $b_{0} \notin D$, and fixing the closed $\operatorname{arcs}\left(\gamma_{1}, \ldots, \gamma_{n} ; \alpha_{1}, \beta_{1}, \ldots, \alpha_{g}, \beta_{g}\right)$ as above, the fiber of $H_{n}^{G}\left(Y, b_{0}\right) \rightarrow$ $\left(Y-b_{0}\right)^{(n)}-\Delta$ over $D$ may be identified with the set of all Hurwitz systems, while by Proposition 1.5 and $\S 1.6$ the fiber of $H_{n}^{G}(Y) \rightarrow Y^{(n)}-\Delta$ over $D$ may be identified with the set of equivalence classes $\left[t_{1}, \ldots, t_{n} ; \lambda_{1}, \mu_{1}, \ldots, \lambda_{g}, \mu_{g}\right]$ of Hurwitz systems modulo inner automorphisms of $G$, i.e. $\left(t_{1}, \ldots, \mu_{g}\right)$ is equivalent to $\left(t_{1}^{\prime}, \ldots, \mu_{g}^{\prime}\right)$ if there exists an element $g \in G$ such that $t_{i}^{\prime}=g t_{i} g^{-1}, \lambda_{k}^{\prime}=$ $g \lambda_{k} g^{-1}, \mu_{k}^{\prime}=g \mu_{k} g^{-1}$ for $\forall i, k$.

An equivalent way of constructing a standard system of closed arcs is the following. One chooses first the $2 g$ simple closed arcs $\alpha_{1}, \beta_{1}, \ldots, \alpha_{g}, \beta_{g}$. Then one chooses $n$ simple arcs which start at $b_{0}$, lie inside the $4 g$-polygon, do not meet outside $b_{0}$, and have for end points the $n$ points of $D$. One enumerates these arcs according to the directions of departure in counterclockwise order. 
The obtained $(n+2 g)$-tuple $\left(\ell_{1}, \ldots, \ell_{n} ; \alpha_{1}, \beta_{1}, \ldots, \alpha_{g}, \beta_{g}\right)$ is called an arc system (cf. [Lo] p.416). One considers the induced ordering of the points of $D$. One can take for $R$ a star-like region which contains the union $\ell_{1} \cup \ldots \cup \ell_{n}$ and let $L=\partial R$. In this way one obtains all ingredients used to construct a standard system of closed arcs $\left(\gamma_{1}, \ldots, \gamma_{n} ; \alpha_{1}, \beta_{1}, \ldots, \alpha_{g}, \beta_{g}\right)$.

2.2. The connected components of $H_{n}^{G}(Y)$ are in one-to-one correspondence with the orbits of the full $n$-strand braid group $\pi_{1}\left(Y^{(n)}-\Delta, D\right)$ acting on the fiber of the topological covering $H_{n}^{G}(Y) \rightarrow Y^{(n)}-\Delta$ over $D$. Similar statement holds about $H_{n}^{G}\left(Y, b_{0}\right) \rightarrow\left(Y-y_{0}\right)^{(n)}-\Delta$ where one considers the braid group $\pi_{1}\left(\left(Y-b_{0}\right)^{(n)}-\Delta, D\right)$. The identification of these fibers with the Hurwitz systems reduces the problem of determining the connected components of $H_{n}^{G}\left(Y, b_{0}\right)$ and $H_{n}^{G}(Y)$ to calculating the action of the respective braid groups on Hurwitz systems and then finding the orbits. Abusing notation we will identify, using Proposition 1.3, the $G$-equivalence classes $\left[C \rightarrow Y, z_{0}\right]$ with the corresponding points $(D, m) \in H_{n}^{G}\left(Y, b_{0}\right)$ and similarly by Proposition 1.5 the $G$-equivalence classes $[C \rightarrow Y]$ with the corresponding points $(D, \underline{m}) \in H_{n}^{G}(Y)$.

Let $\gamma_{i}, \alpha_{k}, \beta_{k}, 1 \leq i \leq n, 1 \leq k \leq g$ be a standard system of closed arcs obtained from an arc system $\ell_{i}, \alpha_{k}, \beta_{k}, 1 \leq i \leq n, 1 \leq k \leq g$ as in $\S 2.1$. Let $D^{u}, 0 \leq u \leq 1$ be a closed arc in $\left(Y-b_{0}\right)^{(n)}-\Delta$ with $D^{0}=D^{1}=D$. We show later, in the cases we need, that starting from the given arc system, one can extend the map $u \mapsto D^{u}$ to a homotopy of arc systems $\ell_{i}^{u}, \alpha_{k}^{u}, \beta_{k}^{u}, 1 \leq i \leq n, 1 \leq k \leq g$ based at $b_{0}$. This yields a corresponding homotopy of closed $\operatorname{arcs} \gamma_{i}^{u}, \alpha_{k}^{u}, \beta_{k}^{u} 1 \leq$ $i \leq n, 1 \leq k \leq g$ which form a standard system for each $u \in[0,1]$. Let $\left[C \rightarrow Y, z_{0}\right]$ correspond to $(D, m) \in H_{n}^{G}\left(Y, b_{0}\right)$ and let $\left(t_{1}, \ldots, t_{n} ; \lambda_{1}, \mu_{1}, \ldots, \lambda_{g}, \mu_{g}\right)$ be the corresponding Hurwitz system. Then the lifting of the closed arc $D^{u}, 0 \leq u \leq 1$ starting from $\left[C \rightarrow Y, z_{0}\right]$ equals, according to Proposition 1.8, to $\left(D^{u}, m^{u}\right.$ : $\left.\pi_{1}\left(Y-D^{u}, b_{0}\right) \rightarrow G\right)$ where

$$
m^{u}\left(\gamma_{i}^{u}\right)=t_{i}, \quad m^{u}\left(\alpha_{k}^{u}\right)=\lambda_{k}, \quad m^{u}\left(\beta_{k}^{u}\right)=\mu_{k}
$$

Let $\gamma_{i}^{1}=\gamma_{i}^{\prime}, \alpha_{k}^{1}=\alpha_{k}^{\prime}, \beta_{k}^{1}=\beta_{k}^{\prime}$ and $m^{1}=m^{\prime}$. We obtain that the end point of the lifting of $D^{u}$ is $\left[C^{\prime} \rightarrow Y, z_{0}^{\prime}\right]$ where $m_{z_{0}^{\prime}}=m^{\prime}: \pi_{1}\left(Y-D, b_{0}\right) \rightarrow G$ is defined by

$$
m^{\prime}\left(\gamma_{i}^{\prime}\right)=t_{i}, \quad m^{\prime}\left(\alpha_{k}^{\prime}\right)=\lambda_{k}, \quad m^{\prime}\left(\beta_{k}^{\prime}\right)=\mu_{k}
$$

Evaluating $m^{\prime}$ at $\gamma_{i}, \alpha_{k}, \beta_{k}$ we obtain

$$
m^{\prime}\left(\gamma_{i}\right)=t_{i}^{\prime}, \quad m^{\prime}\left(\alpha_{k}\right)=\lambda_{k}^{\prime}, \quad m^{\prime}\left(\beta_{k}\right)=\mu_{k}^{\prime} .
$$


So $\left(t_{1}^{\prime}, \ldots, t_{n}^{\prime} ; \lambda_{1}^{\prime}, \mu_{1}^{\prime}, \ldots, \lambda_{g}^{\prime}, \mu_{g}^{\prime}\right)$ is the Hurwitz system corresponding to $\left[C^{\prime} \rightarrow Y, z_{0}^{\prime}\right]$. One may also consider

$$
m\left(\gamma_{i}^{\prime}\right)=t_{i}^{\prime \prime}, \quad m\left(\alpha_{k}^{\prime}\right)=\lambda_{k}^{\prime \prime}, \quad m\left(\beta_{k}^{\prime}\right)=\mu_{k}^{\prime \prime} .
$$

The Hurwitz system $\left(t_{1}^{\prime \prime}, \ldots, t_{n}^{\prime \prime} ; \lambda_{1}^{\prime \prime}, \mu_{1}^{\prime \prime}, \ldots, \lambda_{g}^{\prime \prime}, \mu_{g}^{\prime \prime}\right)$ corresponds to a pair $\left[C^{\prime \prime} \rightarrow Y, z_{0}^{\prime \prime}\right]$ such that $m_{z_{0}^{\prime \prime}}=m^{\prime \prime}: \pi_{1}\left(Y-D, b_{0}\right) \rightarrow G$ satisfies

$$
m^{\prime \prime}\left(\gamma_{i}\right)=t_{i}^{\prime \prime}, \quad m^{\prime \prime}\left(\alpha_{k}\right)=\lambda_{k}^{\prime \prime}, \quad m^{\prime \prime}\left(\beta_{k}\right)=\mu_{k}^{\prime \prime}
$$

Lemma 2.3. Given the topological covering $H_{n}^{G}\left(Y, b_{0}\right) \rightarrow\left(Y-y_{0}\right)^{(n)}-\Delta$ let us lift the arc $D^{1-u}, 0 \leq u \leq 1$ starting from $\left[C \rightarrow Y, z_{0}\right]$. Then the end point is $\left[C^{\prime \prime} \rightarrow Y, z_{0}^{\prime \prime}\right]$.

Proof. In terms of $\gamma_{i}^{\prime}, \alpha_{k}^{\prime}, \beta_{k}^{\prime}$ the monodromy homomorphism of $\left[C \rightarrow Y, z_{0}\right]$ is given by $m\left(\gamma_{i}^{\prime}\right)=t_{i}^{\prime \prime}, m\left(\alpha_{k}^{\prime}\right)=\lambda_{k}^{\prime \prime}, m\left(\beta_{k}^{\prime}\right)=\mu_{k}^{\prime \prime}$. Let us consider the homotopy of closed $\operatorname{arcs} \gamma_{i}^{1-u}, \alpha_{k}^{1-u}, \beta_{k}^{1-u}, 0 \leq u \leq 1$ and the arc in $H_{n}^{G}\left(Y, b_{0}\right)$ given by $n^{u}: \pi_{1}\left(Y-D^{1-u}, b_{0}\right) \rightarrow S_{d}$ where $n^{u}\left(\gamma_{i}^{1-u}\right)=t_{i}^{\prime \prime}, n^{u}\left(\alpha_{k}^{1-u}\right)=\lambda_{k}^{\prime \prime}, n^{u}\left(\beta_{k}^{1-u}\right)=\mu_{k}^{\prime \prime}$. Then $n^{0}=m, n^{1}=m^{\prime \prime}$ (cf. Eq.(8))

Definition 6. Given a closed arc $D^{u}, 0 \leq u \leq 1$ in the configuration space $\left(Y-b_{0}\right)^{(n)}-\Delta$ the transformation of Hurwitz system$\mathrm{s}\left(t_{1}, \ldots, t_{n} ; \lambda_{1}, \mu_{1}, \ldots, \lambda_{g}, \mu_{g}\right) \mapsto\left(t_{1}^{\prime}, \ldots, t_{n}^{\prime} ; \lambda_{1}^{\prime}, \mu_{1}^{\prime}, \ldots, \lambda_{g}^{\prime}, \mu_{g}^{\prime}\right)$ given by Eq.(6) is called a braid move of the first type. The transformation $\left(t_{1}, \ldots, t_{n} ; \lambda_{1}, \mu_{1}, \ldots, \lambda_{g}, \mu_{g}\right) \mapsto\left(t_{1}^{\prime \prime}, \ldots, t_{n}^{\prime \prime} ; \lambda_{1}^{\prime \prime}, \mu_{1}^{\prime \prime}, \ldots, \lambda_{g}^{\prime \prime}, \mu_{g}^{\prime \prime}\right)$ given by $(8)$ is called a braid move of the second type.

The braid moves of the first and of the second type are inverse to each other according to Lemma 2.3. It is evident that the braid moves of both types commute with inner automorphisms of $G$. So the braid moves are well-defined on equivalence classes of Hurwitz systems (cf. $\S 2.1$ ).

2.4. There is a convenient system of generators of $\pi_{1}\left(\left(Y-b_{0}\right)^{(n)}-\Delta, D\right)$. We include here some material borrowed from $[\mathrm{Bi}]$ and $[\mathrm{Sc}]$ for the sake of convenience of the reader and since our choices differ slightly from theirs. Consider the Galois covering $p:\left(Y-b_{0}\right)^{n} \rightarrow\left(Y-b_{0}\right)^{(n)}$ with Galois group $S_{n}$. Restricting to the complement of $\Delta$ one obtains an unramified Galois covering $p:\left(Y-b_{0}\right)^{n}-$ $p^{-1}(\Delta) \rightarrow\left(Y-b_{0}\right)^{(n)}-\Delta$. Following the notation of [FN] if $Q_{1}=\left\{b_{0}\right\}$ one 
denotes $\left(Y-b_{0}\right)^{n}-p^{-1}(\Delta)$ by $F_{1, n} Y$. Let $D=\left\{b_{1}, \ldots, b_{n}\right\}, \tilde{D}=\left(b_{1}, \ldots, b_{n}\right)$. One has an exact sequence

$$
1 \longrightarrow \pi_{1}\left(F_{1, n} Y, \tilde{D}\right) \longrightarrow \pi_{1}\left(\left(Y-b_{0}\right)^{(n)}-\Delta, D\right) \longrightarrow S_{n} \longrightarrow 1
$$

One determines first a system of generators of the pure braid group $\pi_{1}\left(F_{1, n} Y, \tilde{D}\right)$ as follows. Consider the closed arcs in $F_{1, n} Y$ defined by $\left(b_{1}, \ldots, b_{i-1}, r_{i k}(t), b_{i+1}, \ldots, b_{n}\right)$ and $\left(b_{1}, \ldots, b_{i-1}, t_{i k}(t), b_{i+1}, \ldots, b_{n}\right)$, with $t \in$ $[0,1]$, where $r_{i k}$ and $t_{i k}$ are the closed simple arcs based at $b_{i}$ and pictured on Figure 2 by a continuous line and by a dotted line respectively. We denote the

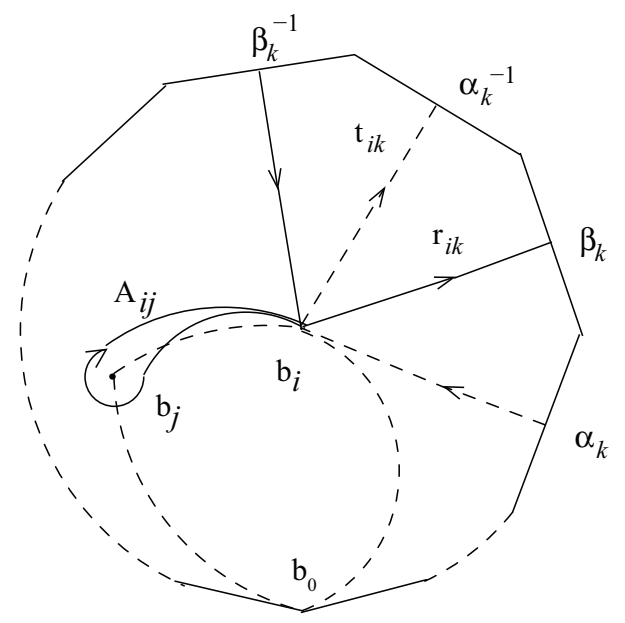

FiguRE 2. Generators of the pure braid group $\pi_{1}\left(F_{1, n}, \tilde{D}\right)$

corresponding homotopy classes by $\rho_{i k}, \tau_{i k} \in \pi_{1}\left(F_{1, n}(Y), \tilde{D}\right)$. Informally $\rho_{i k}$ corresponds to a loop of the $i$-th point along $\alpha_{k}$ and $\tau_{i k}$ corresponds to a loop of the $i$-th point along $\beta_{k}$. Let us denote by $A_{i j}, i<j$ the element of $\pi_{1}\left(F_{1, n} Y, \tilde{D}\right)$ represented by a closed simple arc in $Y^{n}$ which leaves fixed $b_{k}$ for $k \neq i$ and moves $b_{i}$ along the arc pictured on Figure 2. For every $i$ and every $j>i$ the pictured loop is chosen so as to stay on the left of all arcs used to construct $\rho_{i k}$ and $\tau_{i k}$ for $k=1, \ldots, g$.

Claim. The pure braid group $\pi_{1}\left(F_{1, n} Y, \tilde{D}\right)$ is generated by $\rho_{i k}, \tau_{i k}$ and $A_{i j}$ where $i, j=1, \ldots, n, \quad i<j$ and $k=1, \ldots, g$.

Proof. This is proved by induction on $n$. When $n=1$ the claim is obvious. Let $n \geq 2$. Consider the fibration $F_{1, n} Y \rightarrow F_{1, n-1} Y$ defined by $\left(y_{1}, y_{2}, \ldots, y_{n}\right) \rightarrow$ 
$\left(y_{2}, \ldots, y_{n}\right)$. One has an exact sequence

$$
\begin{aligned}
1 \rightarrow \pi_{1}\left(Y-\left\{b_{0}, b_{2}, \ldots, b_{n}\right\}, b_{1}\right) \rightarrow \pi_{1}\left(F_{1, n} Y,\right. & \left.\left(b_{1}, \ldots, b_{n}\right)\right) \\
& \rightarrow \pi_{1}\left(F_{1, n-1} Y,\left(b_{2}, \ldots, b_{n}\right)\right) \rightarrow 1
\end{aligned}
$$

since $\pi_{2}\left(F_{1, n-1} Y\right)=1$ by [FN], Corollary 2.2. The elements $\rho_{i k}, \tau_{i k}, A_{i j}$ with $i \geq 2$ map to the corresponding elements in $\pi_{1}\left(F_{1, n-1} Y,\left(b_{2}, \ldots, b_{n}\right)\right)$. The group $\pi_{1}\left(Y-\left\{b_{0}, b_{2}, \ldots, b_{n}\right\}, b_{1}\right)$ is freely generated by elements which map into $\rho_{1, k}, \tau_{1, k}, A_{1, j}$ where $j \geq 2, \quad k=1, \ldots, g$. This shows the claim.

Following [Fu] p.547 and using the notation of $\S 2.1$ let us denote by $R_{i}$ the simply connected region of $R$ enclosed by the $\operatorname{arcs} \ell_{i}$ and $\ell_{i+1}$ and the $\operatorname{arc}$ of $L$ from $b_{i}$ to $b_{i+1}$. For every $i=1, \ldots, n-1$ choose simple arcs $\eta_{i}:[0,1] \rightarrow Y$ going from $b_{i}$ to $b_{i+1}$ in $R_{i}$ and $\eta_{i}^{\prime}:[0,1] \rightarrow Y$ going from $b_{i+1}$ to $b_{i}$ in $R^{\prime}$. Let $s_{i}:[0,1] \rightarrow Y^{(n)}-\Delta$ be the closed arc

$$
s_{i}(t)=\left\{b_{1}, \ldots, b_{i-1}, \eta_{i}(t), \eta_{i}^{\prime}(t), b_{i+2}, \ldots, b_{n}\right\}
$$

The homotopy class of $s_{i}$ is denoted by $\sigma_{i}$. We may consider $\pi_{1}\left(F_{1, n}(Y), \tilde{D}\right)$ as embedded in $\pi_{1}\left(\left(Y-b_{0}\right)^{(n)}-\Delta, D\right)$. The following relations are easy to verify (cf. [FB] p.249)

$$
\begin{aligned}
& \rho_{i+1, k}=\sigma_{i} \rho_{i k} \sigma_{i}^{-1}, \quad \tau_{i+1, k}=\sigma_{i} \tau_{i k} \sigma_{i}^{-1} \\
& A_{i j}=\sigma_{i}^{-1} \cdots \sigma_{j-2}^{-1} \sigma_{j-1}^{2} \sigma_{j-2} \cdots \sigma_{i}
\end{aligned}
$$

Proposition 2.5 (Birman). Let $a, b \in \mathbb{Z}, \quad 1 \leq a, b \leq n$. The braid group $\pi_{1}\left(\left(Y-b_{0}\right)^{(n)}-\Delta, D\right)$ is generated by $\sigma_{j}, \rho_{a k}, \tau_{b k}$ where $1 \leq j \leq n-1, \quad 1 \leq k \leq g$. The corresponding homotopy classes generate $\pi_{1}\left(Y^{(n)}-\Delta, D\right)$ as well.

Proof. Let us consider the exact sequence (9). The braids $\sigma_{j}$ map to the transpositions $(j j+1), \quad j=1, \ldots, n-1$ which generate $S_{n}$. So $\sigma_{j}, \rho_{i k}, \tau_{i k}, A_{i j}$ with $i<j$ generate $\pi_{1}\left(\left(Y-b_{0}\right)^{(n)}-\Delta, D\right)$ according to the claim proved in $\S 2.4$. The relations (10) show that the generators may be reduced as stated in the theorem. The last statement follows from the surjection $\pi_{1}\left(\left(Y-b_{0}\right)^{(n)}-\Delta, D\right) \rightarrow$ $\pi_{1}\left(Y^{(n)}-\Delta, D\right)$.

We described in $\S 2.4$ closed $\operatorname{arcs}$ in $\left(Y-b_{0}\right)^{(n)}-\Delta$ based at $D=\left\{b_{1}, \ldots, b_{n}\right\}$ whose homotopy classes form a system of generators $\sigma_{j}, \rho_{i k}, \tau_{i k}$ for $\pi_{1}\left(\left(Y-b_{0}\right)^{(n)}-\right.$ $\Delta, D)$. Our aim now is for each of these to construct a homotopy of the standard 
system of closed $\operatorname{arcs} \gamma_{i}^{u}, \alpha_{k}^{u}, \beta_{k}^{u}$ as in $\S 2.2$. This will permit us to calculate eventually the corresponding braid moves of the Hurwitz systems. The calculation of the braid moves $\sigma_{j}, j=1, \ldots, n-1$ is due to Hurwitz (cf. [Hu] or e.g. [Vo2], Theorem 10.3). We define closed $\operatorname{arcs} \delta_{k}:[0,1] \rightarrow Y, \delta_{k}(0)=\delta_{k}(1)=b_{0}, k=0,1, \ldots, g$ as follows. We let $\delta_{0}(t)=b_{0}, \forall t \in[0,1]$. We connect the initial vertex of $\alpha_{1}$ with the end vertex of $\beta_{1}^{-1}$ in the $4 g$-polygon of Figure 1 by a simple arc which belongs to the region $R^{\prime}$ on the right of $L$ (cf. 2.1). This yields $\delta_{1}$. We connect the initial vertex of $\alpha_{1}$ with the end vertex of $\beta_{2}^{-1}$ by a simple arc which belongs to the region on the right of $L$ and on the left of $\delta_{1}$. We denote the corresponding closed arc of $Y$ by $\delta_{2}$. Continuing in this way we obtain $\delta_{1}, \delta_{2}, \ldots, \delta_{g}$ (see Figure 3 ). Clearly $\delta_{k} \simeq\left[\alpha_{1}, \beta_{1}\right] \cdots\left[\alpha_{k}, \beta_{k}\right]$ in $Y-D$ and $\delta_{g} \simeq \gamma_{1} \cdots \gamma_{n}$ according to (5).

Theorem 2.6. Let $Y$ be a compact, closed Riemann surface of genus $g(Y) \geq 1$. Let $b_{0} \in Y$ and let $\gamma_{i}, \alpha_{k}, \beta_{k}$ with $1 \leq i \leq n, 1 \leq k \leq g$ be a standard system of closed arcs as in 2.1. Let $\delta_{k}, k=0,1, \ldots, g$ be the closed arcs defined above. For each of the closed arcs in $\left(Y-b_{0}\right)^{(n)}-\Delta$ constructed in $\S 2.4$ and representing $\sigma_{j}, \rho_{i k}, \tau_{i k}$ there is a homotopy $\gamma_{i}^{u}, \alpha_{k}^{u}, \beta_{k}^{u}, u \in[0,1]$ of the standard system of closed arcs such that the end system $\gamma_{i}^{\prime}, \alpha_{k}^{\prime}, \beta_{k}^{\prime}, 1 \leq i \leq n, 1 \leq k \leq g$ is homotopic to:

a. for $\sigma_{j}$ where $1 \leq j \leq n-1$

$$
\begin{gathered}
\gamma_{i}^{\prime} \simeq \gamma_{i} \quad \text { for } \forall i \neq j, j+1, \quad \alpha_{k}^{\prime} \simeq \alpha_{k}, \beta_{k}^{\prime} \simeq \beta_{k} \quad \text { for } \forall k \\
\gamma_{j}^{\prime} \simeq \gamma_{j+1}, \quad \gamma_{j+1}^{\prime} \simeq \gamma_{j+1}^{-1} \gamma_{j} \gamma_{j+1}
\end{gathered}
$$

b. for $\rho_{i k}$ where $1 \leq i \leq n, 1 \leq k \leq g$

$$
\begin{gathered}
\gamma_{j}^{\prime} \simeq \gamma_{j} \quad \text { for } \forall j \neq i, \quad \alpha_{\ell}^{\prime} \simeq \alpha_{\ell} \quad \text { for } \forall \ell, \quad \beta_{\ell}^{\prime} \simeq \beta_{\ell} \quad \text { for } \forall \ell \neq k \\
\gamma_{i}^{\prime} \simeq \mu_{i k} \gamma_{i} \mu_{i k}^{-1}, \quad \beta_{k}^{\prime} \simeq\left(\zeta_{i k} \gamma_{i} \zeta_{i k}^{-1}\right) \beta_{k}, \quad \text { where } \\
\mu_{i k} \simeq\left(\gamma_{1} \cdots \gamma_{i-1}\right)^{-1} \delta_{k-1} \alpha_{k}\left(\delta_{k}^{-1} \delta_{g}\right)\left(\gamma_{i+1} \cdots \gamma_{n}\right)^{-1}, \quad \zeta_{i k} \simeq\left(\delta_{k}^{-1} \delta_{g}\right)\left(\gamma_{i+1} \cdots \gamma_{n}\right)^{-1} \\
\text { c. for } \tau_{i k} \text { where } 1 \leq i \leq n, 1 \leq k \leq g
\end{gathered}
$$

$$
\begin{gathered}
\gamma_{j}^{\prime} \simeq \gamma_{j} \quad \text { for } \forall j \neq i, \quad \alpha_{\ell}^{\prime} \simeq \alpha_{\ell} \quad \text { for } \forall \ell \neq k, \quad \beta_{\ell}^{\prime} \simeq \beta_{\ell} \quad \text { for } \forall \ell \\
\gamma_{i}^{\prime} \simeq \nu_{i k} \gamma_{i} \nu_{i k}^{-1}, \quad \alpha_{k}^{\prime} \simeq\left(\xi_{i k} \gamma_{i}^{-1} \xi_{i k}^{-1}\right) \alpha_{k}, \quad \text { where } \\
\nu_{i k} \simeq \gamma_{i+1} \cdots \gamma_{n}\left(\delta_{k}^{-1} \delta_{g}\right)^{-1} \beta_{k} \delta_{k-1}^{-1} \gamma_{1} \cdots \gamma_{i-1}, \quad \xi_{i k} \simeq \delta_{k-1}^{-1} \gamma_{1} \cdots \gamma_{i-1}
\end{gathered}
$$


For each of the inverse closed arcs corresponding to $\sigma_{j}^{-1}, \rho_{i k}^{-1}, \tau_{i k}^{-1}$ there is a corresponding homotopy of the standard system $\gamma_{i}, \alpha_{k}, \beta_{k}, 1 \leq i \leq n, 1 \leq k \leq g$ such that the end system $\gamma_{i}^{\prime \prime}, \alpha_{k}^{\prime \prime}, \beta_{k}^{\prime \prime}, 1 \leq i \leq n, 1 \leq k \leq g$ is homotopic to:

d. for $\sigma_{j}^{-1}$ where $1 \leq j \leq n-1$

$$
\begin{gathered}
\gamma_{i}^{\prime \prime} \simeq \gamma_{i} \quad \text { for } \forall i \neq j, j+1, \quad \alpha_{k}^{\prime \prime} \simeq \alpha_{k}, \beta_{k}^{\prime \prime} \simeq \beta_{k} \quad \text { for } \forall k \\
\gamma_{j}^{\prime \prime} \simeq \gamma_{j} \gamma_{j+1} \gamma_{j}^{-1}, \quad \gamma_{j+1}^{\prime \prime} \simeq \gamma_{j}
\end{gathered}
$$

e. $\quad$ for $\rho_{i k}^{-1}$ where $1 \leq i \leq n, 1 \leq k \leq g$

$$
\begin{gathered}
\gamma_{j}^{\prime \prime} \simeq \gamma_{j} \quad \text { for } \forall j \neq i, \quad \alpha_{\ell}^{\prime \prime} \simeq \alpha_{\ell} \quad \text { for } \forall \ell, \quad \beta_{\ell}^{\prime \prime} \simeq \beta_{\ell} \quad \text { for } \forall \ell \neq k \\
\gamma_{i}^{\prime \prime} \simeq \mu_{i k}^{-1} \gamma_{i} \mu_{i k}, \quad \beta_{k}^{\prime \prime} \simeq\left(\tilde{\zeta}_{i k}^{-1} \gamma_{i}^{-1} \tilde{\zeta}_{i k}\right) \beta_{k}, \quad \text { where } \\
\mu_{i k} \text { is as in (b) and } \tilde{\zeta}_{i k} \simeq\left(\gamma_{1} \cdots \gamma_{i-1}\right)^{-1} \delta_{k-1} \alpha_{k}
\end{gathered}
$$

f. $\quad$ for $\tau_{i k}^{-1}$ where $1 \leq i \leq n, 1 \leq k \leq g$

$$
\begin{gathered}
\gamma_{j}^{\prime \prime} \simeq \gamma_{j} \quad \text { for } \forall j \neq i, \quad \alpha_{\ell}^{\prime \prime} \simeq \alpha_{\ell} \quad \text { for } \forall \ell \neq k, \quad \beta_{\ell}^{\prime \prime} \simeq \beta_{\ell} \quad \text { for } \forall \ell \\
\gamma_{i}^{\prime \prime} \simeq \nu_{i k}^{-1} \gamma_{i} \nu_{i k}, \quad \alpha_{k}^{\prime \prime} \simeq\left(\tilde{\xi}_{i k}^{-1} \gamma_{i} \tilde{\xi}_{i k}\right) \alpha_{k}, \quad \text { where } \\
\nu_{i k} \text { is as in (c) and } \tilde{\xi}_{i k} \simeq\left(\gamma_{i+1} \cdots \gamma_{n}\right)\left(\delta_{k}^{-1} \delta_{g}\right)^{-1} \beta_{k}
\end{gathered}
$$

Proof. (a) Here one moves only $\gamma_{j}$ and $\gamma_{j+1}$. Clearly $\gamma_{j}^{\prime} \simeq \gamma_{j+1}$ and $\gamma_{j} \gamma_{j+1} \simeq$ $\gamma_{j}^{\prime} \gamma_{j+1}^{\prime}$, so $\gamma_{j+1}^{\prime} \simeq \gamma_{j+1}^{-1} \gamma_{j} \gamma_{j+1}$.

(b) The effect of the homotopy of $\gamma_{i}, \alpha_{k}, \beta_{k}$ along $\rho_{i k}$ is pictured on Figure 3. One moves the point $b_{i}$ along the $\operatorname{arc} r_{i k}$ pictured on Figure 2 and together with it deforms the closed arc $\gamma_{i}$. At the moment $\gamma_{i}^{u}$ reaches the side $\beta_{k}$ one deforms also $\beta_{k}$ in order that the condition $\gamma_{i}^{u}$ and $\beta_{k}^{u}$ have no points in common except $b_{0}$ remains valid. None of $\gamma_{j}$ for $j \neq i$, or $\alpha_{\ell}$ for $\forall \ell$, or $\beta_{\ell}$ for $\forall \ell \neq k$ changes in this homotopy. The effect of cutting $Y$ along the closed arcs $\alpha_{1}, \beta_{1}, \ldots, \alpha_{k}, \beta_{k}^{\prime}, \ldots, \alpha_{g}, \beta_{g}$ is the same as to cut a region containing $b_{i}$ from the original $4 g$-polygon and glue it along the side $\beta_{i}$ as described in Figure $3(\mathrm{NW})\left(\mathrm{NW}=\right.$ Northwest). We wish to express $\gamma_{i}^{\prime}$ in terms of the standard system $\gamma_{j}, \alpha_{\ell}, \beta_{\ell}, 1 \leq j \leq n, 1 \leq \ell \leq g$. The closed arc $\gamma_{i}^{\prime}$ is pictured in the original $4 g$-polygon on Figure $3(\mathrm{NE})$. It is clear that $\gamma_{i}^{\prime} \simeq \eta_{i k} \gamma_{i} \eta_{i k}^{-1}$ where $\eta_{i k}$ is the closed arc pictured on Figure $3(\mathrm{SW})$. That $\eta_{i k}$ is homotopic to $\left(\gamma_{1} \cdots \gamma_{i-1}\right)^{-1}\left(\delta_{k-1} \alpha_{k}\right) \delta_{k}^{-1}\left(\gamma_{1} \cdots \gamma_{i-1}\right)$ is evident from Figure 3 (SE). Furthermore $\eta_{i k} \gamma_{i} \eta_{i k}^{-1} \simeq\left(\eta_{i k} \gamma_{i}\right) \gamma_{i}\left(\eta_{i k} \gamma_{i}\right)^{-1}$. Using the relation $\gamma_{1} \cdots \gamma_{n} \simeq \delta_{g}$ we obtain 

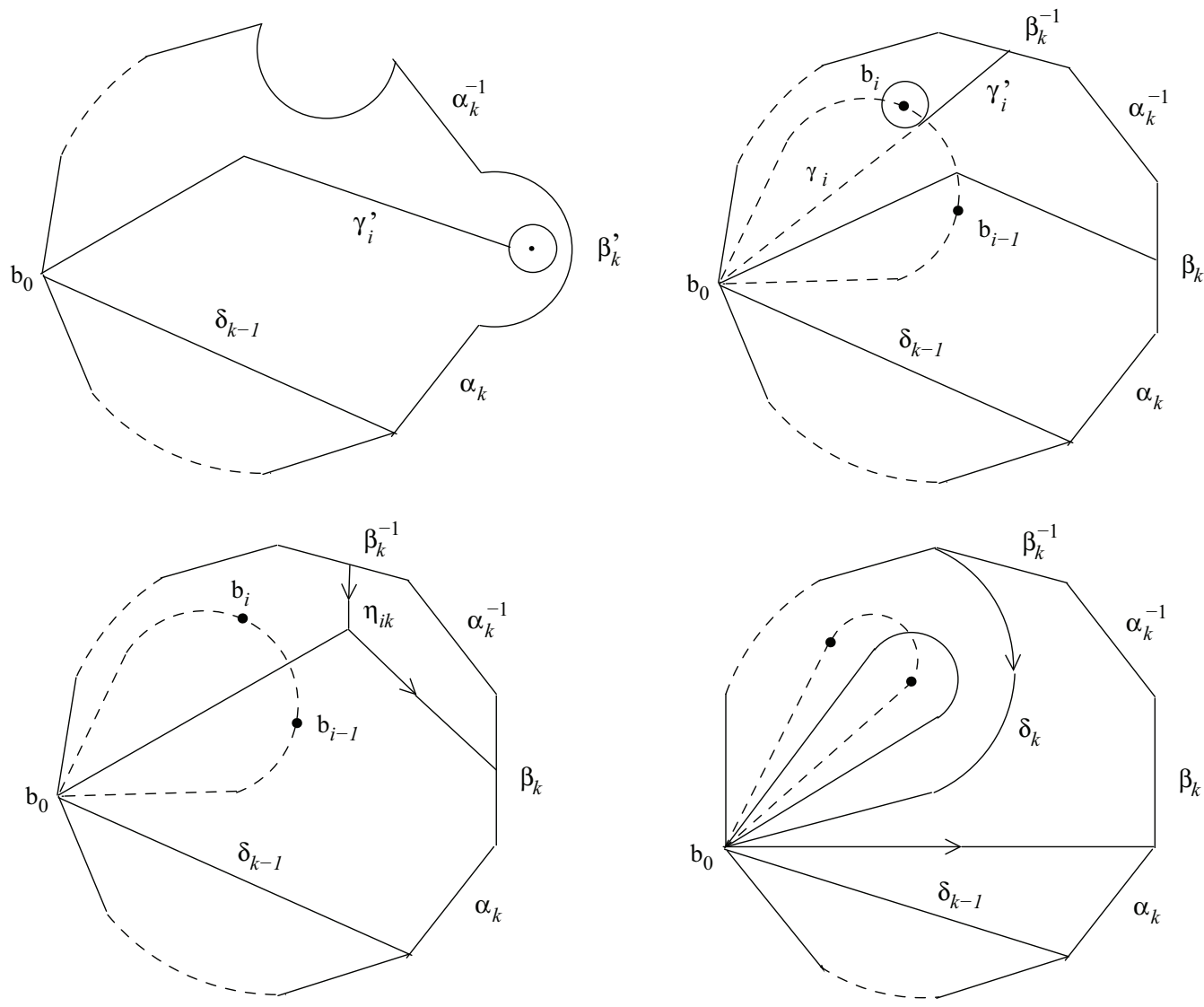

FiguRE 3. Homotopy along $\rho_{i k}$

$\eta_{i k} \gamma_{i} \simeq\left(\gamma_{1} \cdots \gamma_{i-1}\right)^{-1} \delta_{k-1} \alpha_{k}\left(\delta_{k}^{-1} \delta_{g}\right)\left(\gamma_{i+1} \cdots \gamma_{n}\right)^{-1}$. This proves the formula for $\gamma_{i}^{\prime}$ of Part (b). The calculation of $\beta_{k}^{\prime}$ is similar. The closed arc $\beta_{k}^{\prime}$ is pictured in the original $4 g$-polygon on Figure $4(\mathrm{NW})$. It is homotopic to $\omega_{i k} \cdot \beta_{k}$ where $\omega_{i k}$ is the closed arc based at $b_{0}$ pictured on Figure $4(\mathrm{NE})$. We then consider $\delta_{k} \omega_{i k} \delta_{k}^{-1}$ (see Figure $4(\mathrm{SW}))$. The latter is homotopic to $\left(\gamma_{1} \cdots \gamma_{i-1}\right) \gamma_{i}\left(\gamma_{1} \cdots \gamma_{i-1}\right)^{-1}$. We thus obtain $\omega_{i k} \simeq\left(\delta_{k}^{-1} \gamma_{1} \cdots \gamma_{i-1}\right) \gamma_{i}\left(\gamma_{1} \cdots \gamma_{i-1}\right)^{-1} \delta_{k}$. We then have

$$
\begin{aligned}
\omega_{i k} & \simeq \delta_{k}^{-1}\left(\gamma_{1} \cdots \gamma_{i-1} \gamma_{i}\right) \gamma_{i}\left(\gamma_{1} \cdots \gamma_{i-1} \gamma_{i}\right)^{-1} \delta_{k} \\
& \simeq\left[\delta_{k}^{-1} \delta_{g}\left(\gamma_{i+1} \cdots \gamma_{n}\right)^{-1}\right] \gamma_{i}\left[\gamma_{i+1} \cdots \gamma_{n} \delta_{g}^{-1} \delta_{k}\right]
\end{aligned}
$$

since $\gamma_{1} \cdots \gamma_{n} \simeq \delta_{g}$. This proves the second formula of Part (b). 

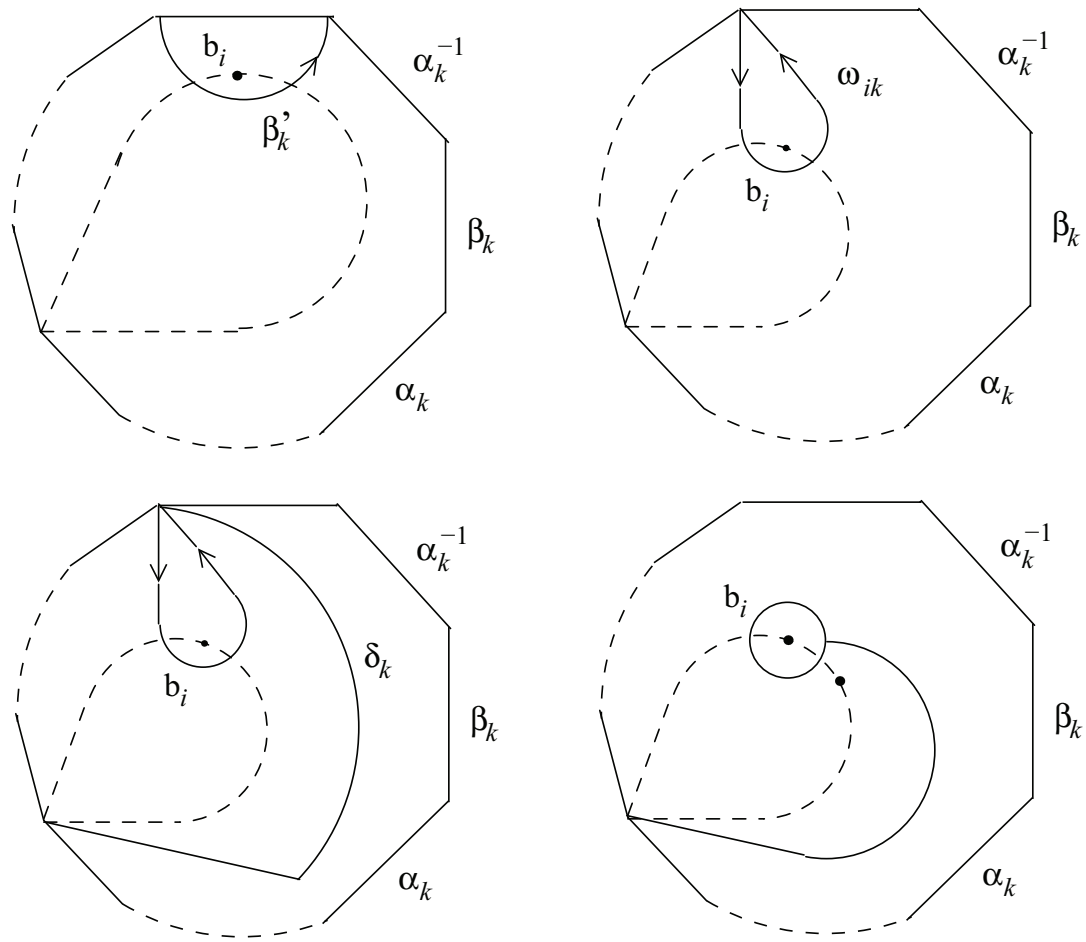

FiguRE 4

(c) The arguments here are very similar to those of Part (b). One deforms $\gamma_{i}$ and $\alpha_{k}$ with $b_{i}^{u}$ moving along the arc $t_{i k}$ (see Figure 2). When $b_{i}^{u}$ returns to $b_{i}$ one obtains closed $\operatorname{arcs} \gamma_{i}^{\prime}$ and $\alpha_{k}^{\prime}$ for which $\gamma_{i}^{\prime} \simeq \theta_{i k} \gamma_{i} \theta_{i k}^{-1}, \alpha_{k}^{\prime}=\varepsilon_{i k} \alpha_{k}$ where $\theta_{i k}$ and $\varepsilon_{i k}$ are represented by arcs in the original $4 g$-polygon pictured on Figure 5 . We calculate $\theta_{i k}$ in the following way. We consider it as a product of four arcs according to the picture. We deform $\theta_{i k}$ in such a way that the first arc becomes a closed simple arc encircling $\left\{b_{1}, \ldots, b_{i}\right\}$ in clockwise direction, then the second arc goes from the initial point of $\alpha_{1}$ to the end point of $\alpha_{k}^{-1}$, the third arc goes from the initial point of $\alpha_{k}$ to the initial point of $\alpha_{1}$ and the fourth arc equals the first one with the opposite orientation. We have accordingly

$$
\theta_{i k} \simeq\left(\gamma_{1} \cdots \gamma_{i}\right)^{-1}\left(\delta_{k} \beta_{k}\right) \delta_{k-1}^{-1}\left(\gamma_{1} \cdots \gamma_{i}\right)
$$

Conjugating $\gamma_{i}$ by $\theta_{i k}$ we may cancel the last factor $\gamma_{i}$ from $\theta_{i k}$ and replace $\left(\gamma_{1} \cdots \gamma_{i}\right)^{-1}$ by $\left(\gamma_{i+1} \cdots \gamma_{n}\right) \delta_{g}^{-1}$. We thus obtain $\gamma_{i}^{\prime}=\nu_{i k} \gamma_{i} \nu_{i k}^{-1}$ where

$$
\nu_{i k} \simeq\left(\gamma_{i+1} \cdots \gamma_{n}\right)\left(\delta_{k}^{-1} \delta_{g}\right)^{-1} \beta_{k} \delta_{k-1}^{-1}\left(\gamma_{1} \cdots \gamma_{i-1}\right)
$$



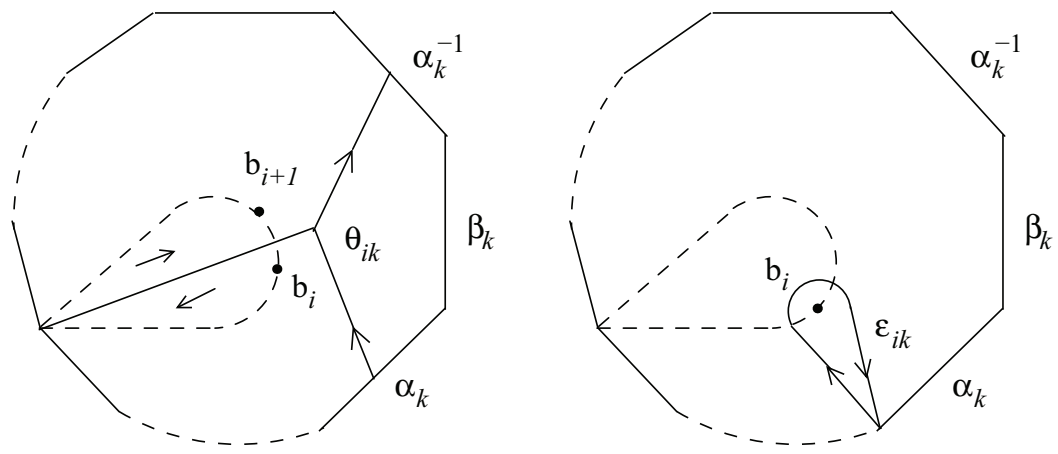

Figure 5

Finally, $\delta_{k-1} \varepsilon_{i k} \delta_{k-1}^{-1} \simeq\left(\gamma_{1} \cdots \gamma_{i-1}\right) \gamma_{i}^{-1}\left(\gamma_{1} \cdots \gamma_{i-1}\right)^{-1}$. This proves the last formula of Part (c).

In order to obtain the formulas of (d), (e) and (f) from those of (a), (b) and (c) we notice that if we apply in each case to $\left(\gamma_{1}^{\prime \prime}, \ldots, \gamma_{n}^{\prime \prime} ; \alpha_{1}^{\prime \prime}, \ldots, \beta_{g}^{\prime \prime}\right)$ the braid moves $\sigma_{j}, \rho_{i k}, \tau_{i k}$ respectively we obtain $\left(\gamma_{1}, \ldots, \gamma_{n} ; \alpha_{1}, \ldots, \beta_{g}\right)$. For example, in order to verify (d) we have $\gamma_{j} \simeq \gamma_{j+1}^{\prime \prime}, \gamma_{j+1} \simeq\left(\gamma_{j+1}^{\prime \prime}\right)^{-1} \gamma_{j}^{\prime \prime} \gamma_{j+1}^{\prime \prime}$. Therefore $\gamma_{j}^{\prime \prime} \simeq \gamma_{j} \gamma_{j+1} \gamma_{j}^{-1}$. In Case (e) applying $\rho_{i k}$ to $\left(\gamma_{1}^{\prime \prime}, \ldots, \beta_{g}^{\prime \prime}\right)$ we obtain $\gamma_{j} \simeq \gamma_{j}^{\prime \prime}$ for $\forall j \neq i, \alpha_{\ell} \simeq \alpha_{\ell}^{\prime \prime}$ for $\forall \ell, \beta_{\ell} \simeq \beta_{\ell}^{\prime \prime}$ for $\forall \ell \neq k$. Furthermore $\gamma_{i} \simeq \mu_{i k}^{\prime \prime} \gamma_{i}^{\prime \prime}\left(\mu_{i k}^{\prime \prime}\right)^{-1}$ where $\mu_{i k}^{\prime \prime}$ is expressed by $\gamma_{j}^{\prime \prime}, \alpha_{\ell}^{\prime \prime}, \beta_{\ell}^{\prime \prime}$ as in (b). Since neither $\gamma_{i}^{\prime \prime}$ nor $\beta_{k}^{\prime \prime}$ enter in this expression we may replace $\gamma_{j}^{\prime \prime}, \alpha_{\ell}^{\prime \prime}, \beta_{\ell}^{\prime \prime}$ by $\gamma_{j}, \alpha_{\ell}, \beta_{\ell}$ and we obtain $\mu_{i k}^{\prime \prime}=\mu_{i k}$. Thus $\gamma_{i}^{\prime \prime} \simeq \mu_{i k}^{-1} \gamma_{i} \mu_{i k}$. Similarly we have $\beta_{k}=\left(\zeta_{i k} \gamma_{i}^{\prime \prime} \zeta_{k}^{-1}\right) \beta_{k}^{\prime \prime}$ where $\zeta_{i k}$ is as in (b). Replacing $\gamma_{i}^{\prime \prime}$ by $\mu_{i k}^{-1} \gamma_{i} \mu_{i k}$ and canceling we obtain $\beta_{k}^{\prime \prime}=\left(\tilde{\zeta}_{i k}^{-1} \gamma_{i}^{-1} \tilde{\zeta}_{i k}\right) \beta_{k}$ where $\tilde{\zeta}_{i k}$ is as in (e). In a similar manner one deduces (f) from (c).

2.7. Recall from Section 1 and $\S 2.1$ that given $D \in Y^{(n)}-\Delta, b_{0} \in Y-D$ and fixing a standard system of closed arcs $\gamma_{1}, \ldots, \gamma_{n} ; \alpha_{1}, \beta_{1}, \ldots, \alpha_{g}, \beta_{g}$ there is a bijective correspondence between the fiber of $H_{n}^{G}\left(Y, b_{0}\right) \rightarrow\left(Y-b_{0}\right)^{(n)}-\Delta$ over $D$ and the set of Hurwitz systems $\left(t_{1}, \ldots, t_{n} ; \lambda_{1}, \mu_{1}, \ldots, \lambda_{g}, \mu_{g}\right)$. Similarly there is a bijective correspondence between the fiber of $U\left(b_{0}\right) \rightarrow\left(Y-b_{0}\right)^{(n)}-\Delta$ and the set of equivalence classes of Hurwitz systems $\left[t_{1}, \ldots, t_{n} ; \lambda_{1}, \mu_{1}, \ldots, \lambda_{g}, \mu_{g}\right]$ modulo inner automorphisms, where $U\left(b_{0}\right)$ is the preimage of $\left(Y-b_{0}\right)^{(n)}-\Delta$ with respect to the map $H_{n}^{G}(Y) \rightarrow Y^{(n)}-\Delta$. In the next theorem we calculate the monodromy action of $\pi_{1}\left(\left(Y-b_{0}\right)^{(n)}-\Delta, D\right)$ on these fibers. According to Proposition 2.5 it suffices to determine the braid moves which correspond to the 
generators $\sigma_{j}, \rho_{i k}, \tau_{i k}$. We denote the corresponding braid moves of the first type (cf. Definition 6) by $\sigma_{j}^{\prime}, \rho_{i k}^{\prime}, \tau_{i k}^{\prime}$ and we denote the corresponding braid moves of the second type (inverse to those of the first type) by $\sigma_{j}^{\prime \prime}, \rho_{i k}^{\prime \prime}, \tau_{i k}^{\prime \prime}$.

Theorem 2.8. Let $\left(t_{1}, \ldots, t_{n} ; \lambda_{1}, \mu_{1}, \ldots, \lambda_{g}, \mu_{g}\right), \lambda_{g+k}=\mu_{k}$, be a Hurwitz system of the group $G$. Let $u_{k}=\left[\lambda_{1}, \mu_{1}\right] \cdots\left[\lambda_{k}, \mu_{k}\right]$ for $k=1, \ldots, g$ and let $u_{0}=1$. The following formulas hold for the braid moves

$$
\left(t_{1}, \ldots, t_{n} ; \lambda_{1}, \mu_{1}, \ldots, \lambda_{g}, \mu_{g}\right) \mapsto\left(t_{1}^{\prime}, \ldots, t_{n}^{\prime} ; \lambda_{1}^{\prime}, \mu_{1}^{\prime}, \ldots, \lambda_{g}^{\prime}, \mu_{g}^{\prime}\right)
$$

of the first type.

a. For $\sigma_{j}^{\prime}$ where $1 \leq j \leq n-1$

$$
t_{i}^{\prime}=t_{i} \quad \text { for } \forall i \neq j, j+1, \quad \lambda_{\ell}^{\prime}=\lambda_{\ell}, \quad \mu_{\ell}^{\prime}=\mu_{\ell} \quad \text { for } \forall \ell
$$

$$
\left(t_{j}, t_{j+1}\right) \mapsto\left(t_{j}^{\prime}, t_{j+1}^{\prime}\right)=\left(t_{j} t_{j+1} t_{j}^{-1}, t_{j}\right)
$$

b. For $\rho_{i k}^{\prime}$ where $1 \leq i \leq n, 1 \leq k \leq g$

$$
\begin{gathered}
t_{j}^{\prime}=t_{j} \quad \text { for } \forall j \neq i, \quad \lambda_{\ell}^{\prime}=\lambda_{\ell} \quad \text { for } \forall \ell, \quad \mu_{\ell}^{\prime}=\mu_{\ell} \quad \text { for } \forall \ell \neq k \\
\left(t_{i}, \mu_{k}\right) \mapsto\left(t_{i}^{\prime}, \mu_{k}^{\prime}\right)=\left(a_{1}^{-1} t_{i} a_{1},\left(b_{1}^{-1} t_{i}^{-1} b_{1}\right) \mu_{k}\right) \quad \text { where } \\
a_{1}=\left(t_{1} \cdots t_{i-1}\right)^{-1} u_{k-1} \lambda_{k}\left(u_{k}^{-1} u_{g}\right)\left(t_{i+1} \cdots t_{n}\right)^{-1}, \quad b_{1}=\left(t_{1} \cdots t_{i-1}\right)^{-1} u_{k-1} \lambda_{k} .
\end{gathered}
$$

c. For $\tau_{i k}^{\prime}$ where $1 \leq i \leq n, 1 \leq k \leq g$

$$
\begin{gathered}
t_{j}^{\prime}=t_{j} \quad \text { for } \forall j \neq i, \quad \lambda_{\ell}^{\prime}=\lambda_{\ell} \quad \text { for } \forall \ell \neq k, \quad \mu_{\ell}^{\prime}=\mu_{\ell} \quad \text { for } \forall \ell \\
\left(t_{i}, \lambda_{k}\right) \mapsto\left(t_{i}^{\prime}, \lambda_{k}^{\prime}\right)=\left(c_{1}^{-1} t_{i} c_{1},\left(d_{1}^{-1} t_{i} d_{1}\right) \lambda_{k}\right) \quad \text { where } \\
c_{1}=t_{i+1} \cdots t_{n}\left(u_{k}^{-1} u_{g}\right)^{-1} \mu_{k}\left(u_{k-1}\right)^{-1} t_{1} \cdots t_{i-1}, \quad d_{1}=t_{i+1} \cdots t_{n}\left(u_{k}^{-1} u_{g}\right)^{-1} \mu_{k} .
\end{gathered}
$$

The following formulas hold for the braid moves

$$
\left(t_{1}, \ldots, t_{n} ; \lambda_{1}, \mu_{1}, \ldots, \lambda_{g}, \mu_{g}\right) \mapsto\left(t_{1}^{\prime \prime}, \ldots, t_{n}^{\prime \prime} ; \lambda_{1}^{\prime \prime}, \mu_{1}^{\prime \prime}, \ldots, \lambda_{g}^{\prime \prime}, \mu_{g}^{\prime \prime}\right)
$$

of the second type.

d. For $\sigma_{j}^{\prime \prime}$ where $1 \leq j \leq n-1$

$$
\begin{gathered}
t_{i}^{\prime \prime}=t_{i} \quad \text { for } \forall i \neq j, j+1, \quad \lambda_{\ell}^{\prime \prime}=\lambda_{\ell}, \quad \mu_{\ell}^{\prime \prime}=\mu_{\ell} \quad \text { for } \forall \ell \\
\left(t_{j}, t_{j+1}\right) \mapsto\left(t_{j}^{\prime \prime}, t_{j+1}^{\prime \prime}\right)=\left(t_{j+1}, t_{j+1}^{-1} t_{j} t_{j+1}\right) .
\end{gathered}
$$


e. For $\rho_{i k}^{\prime \prime}$ where $1 \leq i \leq n, 1 \leq k \leq g$

$$
\begin{gathered}
t_{j}^{\prime \prime}=t_{j} \quad \text { for } \forall j \neq i, \quad \lambda_{\ell}^{\prime \prime}=\lambda_{\ell} \quad \text { for } \forall \ell, \quad \mu_{\ell}^{\prime \prime}=\mu_{\ell} \quad \text { for } \forall \ell \neq k \\
\left(t_{i}, \mu_{k}\right) \mapsto\left(t_{i}^{\prime \prime}, \mu_{k}^{\prime \prime}\right)=\left(a_{2}^{-1} t_{i} a_{2},\left(b_{2}^{-1} t_{i} b_{2}\right) \mu_{k}\right) \quad \text { where } \\
a_{2}=t_{i+1} \cdots t_{n}\left(u_{k}^{-1} u_{g}\right)^{-1} \lambda_{k}^{-1}\left(u_{k-1}\right)^{-1} t_{1} \cdots t_{i-1}, \quad b_{2}=t_{i+1} \cdots t_{n}\left(u_{k}^{-1} u_{g}\right)^{-1} .
\end{gathered}
$$

f. For $\tau_{i k}^{\prime \prime}$ where $1 \leq i \leq n, 1 \leq k \leq g$

$$
\begin{gathered}
t_{j}^{\prime \prime}=t_{j} \quad \text { for } \forall j \neq i, \quad \lambda_{\ell}^{\prime \prime}=\lambda_{\ell} \quad \text { for } \forall \ell \neq k, \quad \mu_{\ell}^{\prime \prime}=\mu_{\ell} \quad \text { for } \forall \ell \\
\left(t_{i}, \lambda_{k}\right) \mapsto\left(t_{i}^{\prime \prime}, \lambda_{k}^{\prime \prime}\right)=\left(c_{2}^{-1} t_{i} c_{2},\left(d_{2}^{-1} t_{i}^{-1} d_{2}\right) \lambda_{k}\right) \text { where } \\
c_{2}=\left(t_{1} \cdots t_{i-1}\right)^{-1} u_{k-1} \mu_{k}^{-1}\left(u_{k}^{-1} u_{g}\right)\left(t_{i+1} \cdots t_{n}\right)^{-1}, \quad d_{2}=\left(t_{1} \cdots t_{i-1}\right)^{-1} u_{k-1} .
\end{gathered}
$$

Proof. Formulas (d), (e) and (f) are obtained from formulas (a), (b) and (c) of Theorem 2.6 respectively applying the homomorphism $m: \pi_{1}\left(Y-D, b_{0}\right) \rightarrow G$ and equalities (7) and (8). By Lemma 2.3 the braid moves of the first type are inverse to the braid moves of the second type. We may thus obtain formulas (a), (b) and (c) from formulas (d), (e) and (f) of Theorem 2.6 respectively applying the homomorphism $m$.

Remark. We notice that the above arguments may be easily adapted to the simpler case $Y \cong \mathbb{P}^{1}, g=0$. Here the only braid moves are $\sigma_{j}^{\prime}$ and $\sigma_{j}^{\prime \prime}, 1 \leq j \leq n-1$, of Theorem 2.8 Part (a) and Part (d).

Corollary 2.9. Using the notation of Theorem 2.8 the following formulas hold.

(i) For $\rho_{i k}^{\prime}$ :

$$
\begin{array}{ll}
\rho_{n k}^{\prime}: t_{n} \mapsto t_{n}^{\prime}=a_{1}^{-1} t_{n} a_{1}, & \text { where } a_{1}=\left(u_{k}^{-1} u_{g}\right)^{-1}\left[\mu_{k}, \lambda_{k}\right] \lambda_{k}\left(u_{k}^{-1} u_{g}\right) \\
\rho_{1 k}^{\prime}: \mu_{k} \mapsto \mu_{k}^{\prime}=\left(b_{1}^{-1} t_{1}^{-1} b_{1}\right) \mu_{k}, & \text { where } b_{1}=u_{k-1} \lambda_{k} \\
\rho_{n k}^{\prime}: \mu_{k} \mapsto \mu_{k}^{\prime}=\left(b_{1}^{-1} t_{n}^{-1} b_{1}\right) \mu_{k}, & \text { where } b_{1}=\left(u_{k}^{-1} u_{g}\right)^{-1}\left[\mu_{k}, \lambda_{k}\right] \lambda_{k} .
\end{array}
$$

(ii) For $\tau_{i k}^{\prime}$ :

$$
\begin{array}{ll}
\tau_{1 k}^{\prime}: t_{1} \mapsto t_{1}^{\prime}=c_{1}^{-1} t_{1} c_{1}, & \text { where } c_{1}=u_{k-1}\left[\lambda_{k}, \mu_{k}\right] \mu_{k} u_{k-1}^{-1} \\
\tau_{1 k}^{\prime}: \lambda_{k} \mapsto \lambda_{k}^{\prime}=\left(d_{1}^{-1} t_{1} d_{1}\right) \lambda_{k}, & \text { where } d_{1}=u_{k-1}\left[\lambda_{k}, \mu_{k}\right] \mu_{k} \\
\tau_{n k}^{\prime}: \lambda_{k} \mapsto \lambda_{k}^{\prime}=\left(d_{1}^{-1} t_{n} d_{1}\right) \lambda_{k}, & \text { where } d_{1}=\left(u_{k}^{-1} u_{g}\right)^{-1} \mu_{k} .
\end{array}
$$


(iii) For $\rho_{i k}^{\prime \prime}$ :

$$
\begin{array}{ll}
\rho_{1 k}^{\prime \prime}: t_{1} \mapsto t_{1}^{\prime \prime}=a_{2}^{-1} t_{1} a_{2}, & \text { where } a_{2}=u_{k-1}\left[\lambda_{k}, \mu_{k}\right] \lambda_{k}^{-1} u_{k-1}^{-1} \\
\rho_{1 k}^{\prime \prime}: \mu_{k} \mapsto \mu_{k}^{\prime \prime}=\left(u_{k}^{-1} t_{1} u_{k}\right) \mu_{k}, & \\
\rho_{n k}^{\prime \prime}: \mu_{k} \mapsto \mu_{k}^{\prime \prime}=\left(b_{1}^{-1} t_{n} b_{1}\right) \mu_{k}, & \text { where } b_{1}=\left(u_{k}^{-1} u_{g}\right)^{-1} .
\end{array}
$$

(iv) For $\tau_{i k}^{\prime \prime}$ :

$$
\begin{array}{rlr}
\tau_{n k}^{\prime \prime}: t_{n} & \mapsto t_{n}^{\prime \prime}=c_{2}^{-1} t_{n} c_{2}, & \text { where } c_{2}=\left(u_{k}^{-1} u_{g}\right)^{-1}\left[\mu_{k}, \lambda_{k}\right] \mu_{k}^{-1}\left(u_{k}^{-1} u_{g}\right) \\
\tau_{1 k}^{\prime \prime}: \lambda_{k} & \mapsto \lambda_{k}^{\prime \prime}=\left(u_{k-1}^{-1} t_{1}^{-1} u_{k-1}\right) \lambda_{k}, & \\
\tau_{n k}^{\prime \prime}: \lambda_{k} & \mapsto \lambda_{k}^{\prime \prime}=\left(d_{2}^{-1} t_{n}^{-1} d_{2}\right) \lambda_{k}, & \text { where } d_{2}=\left(u_{k}^{-1} u_{g}\right)^{-1}\left[\mu_{k}, \lambda_{k}\right] .
\end{array}
$$

In particular

$$
\rho_{n g}^{\prime \prime}: \mu_{g} \mapsto t_{n} \mu_{g}, \quad \tau_{11}^{\prime \prime}: \lambda_{1} \mapsto t_{1}^{-1} \lambda_{1}
$$

Proof. Let us prove the first formula of Part (ii). The other formulas can be either proved similarly or are restatements of particular cases of Theorem 2.8. We have $\tau_{1 k}^{\prime}: t_{1} \mapsto t_{1}^{\prime}=c_{1}^{-1} t_{1} c_{1}$ where $c_{1}=t_{2} \cdots t_{n}\left(u_{k}^{-1} u_{g}\right)^{-1} \mu_{k} u_{k-1}^{-1}$. Since $t_{1} \cdots t_{n}=u_{g}$ it holds $\left(t_{2} \cdots t_{n}\right)^{-1} t_{1}\left(t_{2} \cdots t_{n}\right)=u_{g}^{-1} t_{1} u_{g}$. Hence

$$
\begin{aligned}
t_{1}^{\prime} & =\left(u_{k-1} \mu_{k}^{-1} u_{k}^{-1}\right) t_{1}(\cdots)^{-1}=\left(u_{k-1} \mu_{k}^{-1}\left[\lambda_{k}, \mu_{k}\right]^{-1} u_{k-1}^{-1}\right) t_{1}(\cdots)^{-1} \\
& =\left(u_{k-1}\left[\lambda_{k}, \mu_{k}\right] \mu_{k} u_{k-1}^{-1}\right)^{-1} t_{1}\left(u_{k-1}\left[\lambda_{k}, \mu_{k}\right] \mu_{k} u_{k-1}^{-1}\right)
\end{aligned}
$$

Summing up the discussion made so far in this section we obtain the following result.

Theorem 2.10. Let $n, g$ be integers such that $n>0, g \geq 1$. Let us consider the set of all Hurwitz systems $\left(t_{1}, \ldots, t_{n} ; \lambda_{1}, \mu_{1}, \ldots, \lambda_{g}, \mu_{g}\right)$ of the group $G$ (cf. Definition 5). Let $F$ be the free group generated by the symbols $\sigma_{j}, \rho_{i k}, \tau_{i k}$ where $1 \leq j \leq n-1,1 \leq i \leq n, 1 \leq k \leq g$. Let us consider the action of $F$ on the right on the set of Hurwitz systems of $G$ defined by the formulas for $\sigma_{j}^{\prime}, \rho_{i k}^{\prime}, \tau_{i k}^{\prime}$ of Theorem 2.8 (a)-(c). Then the connected components of $H_{n}^{G}\left(Y, b_{0}\right)$ (cf. Proposition 1.8) correspond bijectively to the orbits of this action and the connected components of $H_{n}^{G}(Y)$ (cf. Proposition 1.9) correspond bijectively to the orbits of the associated action of $F$ on the set of equivalence classes of Hurwitz systems modulo inner automorphisms of $G$. The same statements hold if we 
consider instead the action of $F$ on the left on the set of Hurwitz systems of $G$ defined by the formulas for $\sigma_{j}^{\prime \prime}, \rho_{i k}^{\prime \prime}, \tau_{i k}^{\prime \prime}$ of Theorem $2.8(d)-(f)$.

Proof. $H_{n}^{G}\left(Y, b_{0}\right) \rightarrow\left(Y-b_{0}\right)^{(n)}-\Delta$ is a topological covering map. According to the definition of product of arcs (cf. (1)) the monodromy action of the fundamental group $\pi_{1}\left(\left(Y-b_{0}\right)^{(n)}-\Delta, D\right)$ on the fiber over $D$ is a right action. The identification of this fiber with the set of Hurwitz systems, Proposition 2.5 and the calculation of the braid moves of the first type $\sigma_{j}^{\prime}, \rho_{i k}^{\prime}, \tau_{i k}^{\prime}$ in Theorem 2.8 yield the statement about the orbits of the right action of $F$.

Consider the associated left action $g x=x g^{-1}$. The orbits are the same and according to Lemma 2.3 the braid moves of the second type $\sigma_{j}^{\prime \prime}, \rho_{i k}^{\prime \prime}, \tau_{i k}^{\prime \prime}$ are inverse to those of the first type $\sigma_{j}^{\prime}, \rho_{i k}^{\prime}, \tau_{i k}^{\prime}$ respectively. This shows the statement about the orbits of the left action of $F$.

Let $b_{0} \in Y$. The inclusion $Y-b_{0} \hookrightarrow Y$ induces an epimorphism $\pi_{1}\left(\left(Y-b_{0}\right)^{(n)}-\right.$ $\Delta, D) \rightarrow \pi_{1}\left(Y^{(n)}-\Delta, D\right)$, so the monodromy action of these fundamental groups on the fiber over $D$ of $U\left(b_{0}\right) \rightarrow\left(Y-b_{0}\right)^{(n)}-\Delta($ cf. $\S 2.7)$ and $H_{n}^{G}(Y) \rightarrow Y^{(n)}-\Delta$ respectively yields the same monodromy group. This shows the statement about $H_{n}^{G}(Y)$.

Corollary 2.11. Let $O_{1}, \ldots, O_{k}$ be $k$ different conjugacy classes of $G$. Let $\underline{n}=$ $n_{1} O_{1}+\cdots+n_{k} O_{k}$, let $|\underline{n}|=n_{1}+\cdots+n_{k}=n$ and let $H_{\underline{n}}^{G}\left(Y, y_{0}\right)$ be as in Definition 4. Then $H_{\underline{n}}^{G}\left(Y, y_{0}\right)$ is connected (hence irreducible) if and only if $F$ acts transitively on the set of Hurwitz systems $\left(t_{1}, \ldots, t_{n} ; \lambda_{1}, \mu_{1}, \ldots, \lambda_{g}, \mu_{g}\right)$ which have the property that $n_{i}$ of the elements $t_{1}, \ldots, t_{n}$ belong to $O_{i}, i=1, \ldots, k$. Similarly $H_{\underline{n}}^{G}(Y)$ is connected (hence irreducible) if and only if $F$ acts transitively on the set of equivalence classes of Hurwitz systems $\left[t_{1}, \ldots, t_{n} ; \lambda_{1}, \mu_{1}, \ldots, \lambda_{g}, \mu_{g}\right]$ which have the above property.

\section{HuRWitz SYSTEMS OF ARBITRARY GROUPS}

The definitions and the arguments of Section 1 and Section 2 may be applied to arbitrary, possibly infinite, groups. If $G$ is infinite the sets $H_{n}^{G}\left(Y, y_{0}\right)$ and $H_{n}^{G}(Y)$ are non empty if and only if $G$ has at least one Hurwitz system of generators. In this case each one of $H_{n}^{G}\left(Y, y_{0}\right)$ and $H_{n}^{G}(Y)$ has a structure of a complex manifold, the maps $H_{n}^{G}\left(Y, y_{0}\right) \rightarrow\left(Y-y_{0}\right)^{(n)}-\Delta$ and $H_{n}^{G}(Y) \rightarrow Y^{(n)}-\Delta$ given by $(D, m) \mapsto$ 
$D$ and $(D, \underline{m}) \mapsto D$ respectively, are topological covering maps and are holomorphic. The set $H_{n}^{G}\left(Y, y_{0}\right)$ corresponds bijectively to the set of $G$-equivalence classes $\left[p^{\prime}: C^{\prime} \rightarrow Y-D, z_{0}\right]$ where:

- $D \in Y^{(n)}-\Delta, \quad y_{0} \notin D$;

- $p^{\prime}: C^{\prime} \rightarrow Y-D$ is a holomorphic map of Riemann surfaces, which is a regular topological covering map;

- an isomorphism $G \stackrel{\sim}{\longrightarrow} \operatorname{Deck}\left(C^{\prime} / Y-D\right)$ is fixed;

- if $D=b_{1}+\cdots+b_{n}$, the local monodromies of $p^{\prime}$ at the points $b_{1}, \ldots, b_{n}$ are all nontrivial;

- $z_{0} \in p^{-1}\left(y_{0}\right)$.

Similarly, the set $H_{n}^{G}(Y)$ corresponds bijectively to the set of $G$-equivalence classes $\left[p^{\prime}: C^{\prime} \rightarrow Y-D\right]$ as above with the conditions $y_{0} \notin D$ and $z_{0} \in p^{\prime-1}\left(y_{0}\right)$ skipped. In no point of the proofs of Theorem 2.8 and Theorem 2.10 the condition that $G$ is finite is used. So they hold under the only assumption that the group $G$ has at least one Hurwitz system of generators $\left(t_{1}, \ldots, t_{n} ; \lambda_{1}, \mu_{1}, \ldots, \lambda_{g}, \mu_{g}\right)$.

Definition 7. We call two Hurwitz systems of $G$ braid-equivalent if one is obtained from the other by a finite sequence of braid moves $\sigma_{j}^{\prime}, \rho_{i k}^{\prime}, \tau_{i k}^{\prime}, \sigma_{j}^{\prime \prime}, \rho_{i k}^{\prime \prime}, \tau_{i k}^{\prime \prime}$ where $1 \leq j \leq n-1,1 \leq i \leq n, 1 \leq k \leq g$. We denote the braid equivalence by $\sim$.

Theorem 2.10 shows that two elements $(D, m)$ and $\left(D, m^{\prime}\right)$ of $H_{n}^{G}\left(Y, y_{0}\right)$ correspond to braid-equivalent Hurwitz systems if and only if they belong to one and the same connected component of $H_{n}^{G}\left(Y, y_{0}\right)$.

The following proposition is a useful tool for determining if two Hurwitz systems are braid-equivalent.

Proposition 3.1. Let $\left(t_{1}, \ldots, t_{n} ; \lambda_{1}, \mu_{1}, \ldots, \lambda_{g}, \mu_{g}\right)$ be a Hurwitz system of the group $G$. Suppose that $t_{i} t_{i+1}=1$. Let $H$ be the subgroup of $G$ generated by $\left\{t_{1}, \ldots, t_{i-1}, t_{i+2}, \ldots, t_{n} ; \lambda_{1}, \mu_{1}, \ldots, \lambda_{g}, \mu_{g}\right\}$. Then for every $h \in H$ the given Hurwitz system is braid-equivalent to $\left(t_{1}, \ldots, t_{i-1}, t_{i}^{h}, t_{i+1}^{h}, t_{i+2}, \ldots, t_{n} ; \lambda_{1}, \mu_{1}, \ldots, \lambda_{g}, \mu_{g}\right)$

Proof. Let us fix $t_{1}, \ldots, t_{i-1}, t_{i+2}, \ldots, t_{n} ; \lambda_{1}, \mu_{1}, \ldots, \lambda_{g}, \mu_{g}$. Let $H_{1} \subseteq H$ be the subset consisting of elements $h$ such that the statement of the lemma holds for an arbitrary pair $\left(t_{i}, t_{i+1}\right)=\left(\tau, \tau^{-1}\right)$. 
Step 1. We claim that $H_{1}$ is a subgroup of $H$. Let $h \in H_{1}$ and let $t=$ $h t_{i} h^{-1}$. Then $t_{i}=t^{h}$, so by assumption $\left(\ldots, t^{h},\left(t^{-1}\right)^{h}, \ldots\right)$ can be obtained from $\left(\ldots, t, t^{-1}, \ldots\right)$ by a sequence of braid moves. Then one can obtain $\left(\ldots, t, t^{-1}, \ldots\right)$ from $\left(\ldots, t_{i}, t_{i}^{-1}, \ldots\right)$ by the inverse sequence of braid moves. Thus $h^{-1} \in H_{1}$. If $h_{1}, h_{2} \in H_{1}$, then $\left(\ldots, t_{i}, t_{i}^{-1}, \ldots\right) \sim\left(\ldots, t_{i}^{h_{1}},\left(t_{i}^{-1}\right)^{h_{1}}, \ldots\right) \sim$ $\left(\ldots, t_{i}^{h_{1} h_{2}},\left(t_{i}^{-1}\right)^{h_{1} h_{2}}, \ldots\right)$, so $h_{1} h_{2} \in H_{1}$.

Step 2. We claim that for every $\ell \neq i, i+1$ the element $t_{\ell}$ belongs to $H_{1}$. Applying a sequence of braid moves $\sigma_{j}^{\prime}, \sigma_{j}^{\prime \prime}$ we can move the adjacent pair $\left(t_{i}, t_{i+1}\right)$ wherever we want among the first $n$ elements of the Hurwitz system without changing the other elements. So, move $\left(t_{i}, t_{i+1}\right)$ to the left side of $t_{\ell}$. Then we have $\left(t_{i}, t_{i+1}, t_{\ell}\right) \sim\left(t_{i}, t_{\ell}, t_{\ell}^{-1} t_{i+1} t_{\ell}\right) \sim\left(t_{\ell}, t_{\ell}^{-1} t_{i} t_{\ell}, t_{\ell}^{-1} t_{i+1} t_{\ell}\right)$. We then move the pair $\left(t_{i}^{h}, t_{i+1}^{h}\right)$ with $h=t_{\ell}$ back to the initial position.

Step 3 . We claim thet for every $k=1, \ldots, g$ the element $h=u_{k-1} \lambda_{k} u_{k}^{-1}$ belongs to $H_{1}$. First suppose that $i=1$. In this case $t_{2}=t_{1}^{-1}$. Let us perform the braid move $\rho_{1 k}^{\prime}$. One obtains $\left(t_{1}, t_{1}^{-1}, \ldots, \lambda_{k}, \mu_{k}, \ldots\right) \sim\left(t_{1}^{\prime}, t_{1}^{-1}, \ldots, \lambda_{k}, \mu_{k}^{\prime}, \ldots\right)$ where $t_{1}^{\prime}=a_{1}^{-1} t_{1} a_{1}$ with $a_{1}=u_{k-1} \lambda_{k}\left(u_{k}^{-1} u_{g}\right)\left(t_{2} \cdots t_{n}\right)^{-1}$ and $\mu_{k}^{\prime}=\left(b_{1}^{-1} t_{1}^{-1} b_{1}\right) \mu_{k}$ with $b_{1}=u_{k-1} \lambda_{k}$. We have $t_{1} \cdots t_{n}=u_{g}$, so $a_{1}=u_{k-1} \lambda_{k} u_{k}^{-1} t_{1}$. Let us move $t_{2}=t_{1}^{-1}$ to the first place using $\sigma_{1}^{\prime \prime}$. One obtains $\left(t_{1}^{-1}, t_{1} t_{1}^{\prime} t_{1}^{-1}, \ldots, \lambda_{k}, \mu_{k}^{\prime}, \ldots\right)$. Here $t_{1} t_{1}^{\prime} t_{1}^{-1}=t_{1}^{h}$ where $h=u_{k-1} \lambda_{k} u_{k}^{-1}$. Let us perform again $\rho_{1 k}^{\prime}$. The elements $u_{k-1}, \lambda_{k}$ have not changed with respect to the original Hurwitz system, so $\mu_{k}^{\prime} \mapsto\left[\left(u_{k-1} \lambda_{k}\right)^{-1}\left(t_{1}^{-1}\right)^{-1}\left(u_{k-1} \lambda_{k}\right)\right] \mu_{k}^{\prime}=\mu_{k}$. Moving the second element $t_{1}^{h}$ to the first place by $\sigma_{1}^{\prime \prime}$ we obtain $\left(t_{1}^{h}, \tilde{t}_{2}, t_{3}, \ldots, \lambda_{k}, \mu_{k}, \ldots\right)$. Since $t_{1} t_{2} t_{3} \cdots t_{n}=u_{g}=t_{1}^{h} \tilde{t}_{2} t_{3} \cdots t_{n}$ we must have $\tilde{t}_{2}=\left(t_{1}^{-1}\right)^{h}$. This proves $\left(t_{1}, t_{1}^{-1}, t_{3}, \ldots\right) \sim\left(t_{1}^{h},\left(t_{1}^{-1}\right)^{h}, t_{3}, \ldots\right)$ with $h=u_{k-1} \lambda_{k} u_{k}^{-1}$. One extends this braid equivalence to every adjacent pair $\left(t_{i}, t_{i+1}\right)$ with $t_{i} t_{i+1}=1$ by moving first the pair to the front, applying the braid equivalence we have just proved and moving the obtained pair back to the original position.

Step 4 . We claim that for every $k=1, \ldots, g$ the element $h=u_{k-1} \mu_{k}^{-1} u_{k}^{-1}$ belongs to $H_{1}$. The proof is the same as that of Step 3. One uses the braid move $\tau_{1 k}^{\prime \prime}$ instead of $\rho_{1 k}^{\prime}$.

Step 5. By the preceding steps it remains to verify that $\lambda_{k}, \mu_{k}$ for $\forall k$ belong to the subgroup $H_{2} \subseteq H_{1}$ generated by $t_{j}, u_{k-1} \lambda_{k} u_{k}^{-1}, u_{k-1} \mu_{k}^{-1} u_{k}^{-1}$ where $j=1, \ldots, i-1, i+2, \ldots, n$ and $k=1, \ldots, g$. We prove this by induction on 
$k$. We have $\lambda_{1} u_{1}^{-1} \in H_{2}, u_{1} \mu_{1}=\left(\mu_{1}^{-1} u_{1}^{-1}\right)^{-1} \in H_{2}$, so $\lambda_{1} \mu_{1} \in H_{2}$. Furthermore $H_{2} \ni u_{1} \mu_{1}=\left[\lambda_{1}, \mu_{1}\right] \mu_{1}=\lambda_{1} \mu_{1} \lambda_{1}^{-1}$. So $\lambda_{1}^{ \pm 1} \in H_{2}$ and $\mu_{1}^{ \pm 1} \in H_{2}$. Let $k \geq 2$. Suppose, by inductive assumption, that $\lambda_{1}, \mu_{1}, \ldots, \lambda_{k-1}, \mu_{k-1}$ belong to $H_{2}$. Then $u_{k-1}=\prod_{\ell=1}^{k-1}\left[\lambda_{\ell}, \mu_{\ell}\right] \in H_{2}$. We have $u_{k-1} \lambda_{k} u_{k}^{-1} \in H_{2}$, $u_{k} \mu_{k} u_{k-1}^{-1}=\left(u_{k-1} \mu_{k}^{-1} u_{k}^{-1}\right)^{-1} \in H_{2}$, so $u_{k-1} \lambda_{k} \mu_{k} u_{k-1}^{-1} \in H_{2}$ and therefore $\lambda_{k} \mu_{k} \in H_{2}$. Furthermore $H_{2} \ni u_{k} \mu_{k} u_{k-1}^{-1}=u_{k-1}\left[\lambda_{k}, \mu_{k}\right] \mu_{k} u_{k-1}^{-1}$. Therefore $\lambda_{k} \mu_{k} \lambda_{k}^{-1} \in H_{2}$, so $\lambda_{k}^{ \pm 1} \in H_{2}$ and $\mu_{k}^{ \pm 1} \in H_{2}$.

The proposition is proved.

Acknowledgements. The author was on leave of absence from the Institute of Mathematics and Informatics of the Bulgarian Academy of Sciences.

\section{REFERENCES}

[BE] I. Berstein and A.L. Edmonds, On the classification of generic branched coverings of surfaces, Illinois Journ. Mathem. 28 (1984), 64-82.

[Bi] J. S. Birman, On braid groups, Comm. Pure Appl. Math. 22 (1968), 41-72.

[CLP] F. Catanese, M. Lönne and F. Perroni, The irreducible components of the moduli space of dihedral covers of algebraic curves, arXiv:1206.5498 [math.AG].

[Cl] A. Clebsch, Zur Theorie der Riemann'schen Fläche, Math. Annalen 6 (1872), 216-230.

[Em] M. Emsalem, Familles de revêtements de la droite projective, Bull. Soc. Math. France 123 (1995), no. 1, 47-85.

[FB] E. Fadell and J. Van Buskirk, The braid groups of $E^{2}$ and $S^{2}$, Duke Math. J. 29 (1962), 243-257.

[FN] E. Fadell and L. Neuwirth, Configuration spaces, Math. Scand. 10 (1962), 111-118.

[Fo] O. Forster, Lectures on Riemann surfaces, Graduate Texts in Mathematics, vol. 81, Springer-Verlag, New York, 1991.

[Fr] M. Fried, Fields of definition of function fields and Hurwitz families-groups as Galois groups, Comm. Algebra 5 (1977), no. 1, 17-82.

[FV] M. D. Fried and H. Völklein, The inverse Galois problem and rational points on moduli spaces, Math. Ann. 290 (1991), no. 4, 771-800.

[Fu] W. Fulton, Hurwitz schemes and irreducibility of moduli of algebraic curves, Ann. of Math. (2) 90 (1969), 542-575.

[SGA1] A. Grothendieck, Revêtements étales et groupe fondamental, Springer-Verlag, Berlin, 1971, Séminaire de Géométrie Algébrique du Bois Marie 1960-1961 (SGA 1), Lecture Notes in Mathematics, Vol. 224.

[HGS] J. Harris, T. Graber, and J. Starr, A note on Hurwitz schemes of covers of a positive genus curve, arXiv:math/0205056 [math.AG].

[Hu] Hurwitz A., Ueber Riemann'schen Flächen mit gegebenen Verzweigungspunkten, Math. Ann. 39 (1891), 1-61. 
[K1] V. Kanev, Irreducibility of Hurwitz spaces, Preprint N. 241, Dipartimento di Matematica e Informatica, Università di Palermo, Feb. 2004, arXiv:math/0509154 [math.AG].

[K2] V. Kanev, Unirationality of Hurwitz spaces of coverings of degree $\leq 5$, Int. Math Res. Notices 2013 (2013), no. 13, 3006-3052.

[KuKh] Vik. S. Kulikov and V. M. Kharlamov, The semigroups of coverings. (Russian), Izv. Ross. Akad. Nauk Ser. Mat. 77 (2013), no. 3, 163-198 (english translation in Izv. Math. 77 (2013), no. 3, 594-626).

[Lo] E. Looijenga, On the tautological ring of $\mathcal{M}_{g}$, Invent. Math. 121 (1995), no. 2, 411-419.

[Ma] W. S. Massey, A basic course in algebraic topology, Graduate Texts in Mathematics, vol. 127, Springer-Verlag, New York, 1991.

[Nar] R. Narasimhan, Several complex variables, Chicago Lectures in Mathematics, University of Chicago Press, Chicago, IL, 1995, Reprint of the 1971 original.

[Sc] G. P. Scott, Braid groups and the group of homeomorphisms of a surface, Proc. Cambridge Philos. Soc. 68 (1970), 605-617.

[Ve1] F. Vetro, Irreducibility of Hurwitz spaces of coverings with one special fiber, Indag. Math. (N.S.) 17 (2006), no. 1, 115-127.

[Ve2] F. Vetro, Irreducibility of Hurwitz spaces of coverings with monodromy groups Weyl groups of type $W\left(B_{d}\right)$, Boll. Unione Mat. Ital. Sez. B Artic. Ric. Mat. (8) 10 (2007), no. $2,405-431$.

[Ve3] F. Vetro, Irreducibility of Hurwitz spaces of coverings with one special fiber and monodromy group a Weyl group of type $D_{d}$, Manuscripta Math. 125 (2008), no. 3, 353-368.

[Ve4] F. Vetro, Coverings with special fibers and the monodromy group $S_{d}$. (Russian), Izv. Ross. Akad. Nauk Ser. Mat., 76 (2012), no. 6, 39-44 (english translation in Izv. Math. 76 (2012), no. 6, 1110-1115).

[Vo1] H. Völklein, Moduli spaces for covers of the Riemann sphere, Israel J. Math. 85 (1994), no. 1-3, 407-430.

[Vo2] H. Völklein, Groups as Galois groups, Cambridge Studies in Advanced Mathematics, vol. 53, Cambridge University Press, Cambridge, 1996.

Vassil Kanev

Dipartamento di Matematica e Informatica

Università di Palermo

Via Archirafi, 34

90123 Palermo

Italy

E-mail: kanev@math.unipa.it 